\title{
High-Velocity Clouds and the Local Intergalactic Medium
}

\author{
Ludwig Biermann Award Lecture
}

Philipp Richter

Argelander-Institut für Astronomie (AIfA), Universität Bonn, Auf dem Hügel 71, 53121 Bonn

prichter@astro.uni-bonn.de

\begin{abstract}
In this article I review recent observations of the gaseous halos of galaxies and the intergalactic medium at low redshift. In the first part I discuss distribution, metal content, and physical properties of the Galactic intermediate- and high-velocity clouds and the hot halo of the Milky Way. Recent absorption and emission measurements show that the Galaxy's tidal interaction with the Magellanic Clouds, the infall of lowmetallicity gas, as well as the circulation of gas as part of the galactic fountain contribute to the observed distribution of gas in the halo of the Milky Way. In the second part of this article I give a short overview on the circumgalactic gaseous environment of other nearby spiral galaxies. Multi-wavelength observations demonstrate that neutral and ionized gaseous halos of galaxies are common, and that they extend deep into intergalactic space. These studies suggest that the gaseous material around spiral galaxies is tightly connected to the on-going hierarchical formation and evolution of these galaxies. In the last part of this article I summarize recent quasar absorption-line measurements of the local intergalactic medium. In accordance with cosmological simulations, absorption-line studies in the far-ultraviolet indicate that both the photoionized Ly $\alpha$ forest and the shock-heated warm-hot intergalactic medium harbor a substantial fraction of the baryons in the local Universe.
\end{abstract}

\section{Introduction}

Studying the distribution and physical properties of low-density gas that is situated in the extended halos of galaxies and in the intergalactic medium is of fundamental importance for our understanding of the formation and evolution of structure in the Universe. Multi-wavelength observations of gaseous halos and circumgalactic gas of galaxies provide a detailed insight into the various complex processes that balance the exchange of gaseous matter and energy between individual galaxies and the intergalactic medium. Supernova explosions in spiral galaxies create large cavities filled with hot gas in the gaseous 
disk. This gas eventually breaks out of the disk and flows into the halo and into intergalactic space, where it enriches the circumgalactic gas and the intergalactic medium with heavy elements. In addition, the interaction and the merging of galaxies transports large amounts of interstellar material into the halos and immediate intergalactic environment of galaxies. Gas that is related to tidal interactions of galaxies therefore indicates the on-going growth and evolution of galaxies in terms of the hierarchical structure-evolution in the Universe. Finally, knowledge about the distribution and physical properties of the intergalactic medium is important to understand the global picture of how large-scale structures form and how the baryonic matter is distributed throughout the Universe.

With the availability of space-based spectroscopic instruments operating in the Ultraviolet and Far-Ultraviolet (UV and FUV, respectively), such as the Orbiting and Retrievable Far and Extreme Ultraviolet Spectrometer (ORFEUS), the Space Telescope Imaging Spectrograph (STIS), and the Far Ultraviolet Spectroscopic Explorer (FUSE) it has become possible to explore the gaseous halos of the Milky Way and other galaxies and the local intergalactic medium in absorption against distant extragalactic UV background sources like quasars (QSOs) and Active Galactic Nuclei (AGN). The UV and FUV spectroscopic range is particularly interesting for studying the lowdensity, multi-phase circumgalactic medium, because many atomic and molecular species and their ions have most of their electronic transitions in the region between 900 and $3000 \AA$ (e.g., $\mathrm{H}_{2}, \mathrm{H}$ I, D I, C II, C III, C IV, N I, O I, O vi, Si II, Si III, and Fe II). Measurements of absorption lines from these species together with supporting emission-line observations in other wavelength ranges therefore allow us to analyze in detail the gas in the halos of galaxies and in the intergalactic medium in all of its phases (i.e., from molecular to highlyionized).

This article is the written version of my Ludwig Biermann Award Lecture that was held in September 2005 at the annual meeting of the Astronomische Gesellschaft in Cologne/Germany. The article is not meant to represent an overall review on high-velocity clouds and the local intergalactic medium, but rather reflects my personal view on this topic together with an overview of my own contributions in this field. This article is organized as follows. In Section 2 I discuss distribution and properties of the intermediate- and highvelocity clouds in the halo of the Milky Way. In Section 3, I review the hot Galactic corona and highly-ionized gas in the circumgalactic environment of the Galaxy. Section 4 deals with circumgalactic gaseous structures in other galaxies. In Secion 5, properties and baryon content of the local intergalactic medium are discussed. A short conclusion is given in Section 6 . 


\section{The Milky Way's High-Velocity Clouds}

\subsection{Overview}

Ever since their detection more than forty years ago, the origin and nature of the intermediate and high-velocity clouds (IVCs and HVCs, respectively) in the halo of the Milky Way has been controversial. These gas clouds are concentrations of neutral hydrogen (H I) with radial velocities that are inconsistent with a simple model of differential galactic rotation. They are thought to be located in the inner and outer halo and nearby intergalactic environment of the Milky Way. The distinction between IVCs and HVCs is loosely based on the observed radial velocities of the clouds; IVCs have radial velocities with respect to the Local Standard of Rest (LSR) of $30 \mathrm{~km} \mathrm{~s}^{-1} \leq\left|V_{\mathrm{LSR}}\right| \leq 90$ $\mathrm{km} \mathrm{s}^{-1}$, typically, while HVCs have typical velocities $\left|V_{\mathrm{LSR}}\right|>90 \mathrm{~km} \mathrm{~s}^{-1}$.

This extraplanar neutral gas with high radial velocities was first detected in optical absorption spectra of stars at high galactic latitudes (Münch 1952; Münch \& Zirin 1961). Based on these observations, Spitzer (1956) predicted that the neutral halo clouds must be embedded in a Corona of hot gas that provides the thermal pressure necessary to to keep the clouds together (see Section 3). The first detection of the IVCs and HVCs in $\mathrm{H} \mathrm{I} 21 \mathrm{~cm}$ radio emission was reported some years later by Muller et al. (1963). Oort (1970) proposed that these clouds represent condensed gaseous relicts from the formation phase of the Milky Way. This idea later was revived by Blitz et al. (1999), who suggested that HVCs represent the building blocks of galaxies in a hierarchical galaxy formation scenario. Since the Galaxy is surrounded by smaller satellite galaxies (e.g., the Magellanic Clouds), another explanation is that IVCs and HVCs are gaseous streams related to the merging and accretion of these satellites by the Milky Way. In this picture, HVCs would be the gaseous counterparts of the Milky Way's circumgalactic stellar streams, which are believed to represent the relics of dwarf galaxies that have been accreted by the Milky Way (e.g., Ibata 1994). While all these models assume that HVCs are truly extragalactic objects that are about to merge with the Galaxy from outside, there are other scenarios that see the IVCs and HVCs as objects that have their origin in the disk of the Milky Way, e.g., as part of the so-called "galactic fountain". In the galactic fountain model (Shapiro \& Field 1976; Houck \& Bregman 1990), hot gas is ejected out of the Galactic disk by supernova explosions, and part of this gas falls back in the form of condensed neutral clouds that move at intermediate and high radial velocities. Whatever the origin of the Milky Way's IVCs and HVCs is, it has become clear that they must play an important role in the evolution of our Galaxy.

Two extremely important parameters to distinguish between the Galactic and extragalactic models of IVCs and HVCs are the distance and the chemical composition of these clouds. Both distance and metal abundance measurements require the use of high-resolution absorption line spectra. Consequently, absorption line measurements in the FUV and in the optical have been used extensively during the last few years to obtain new information 


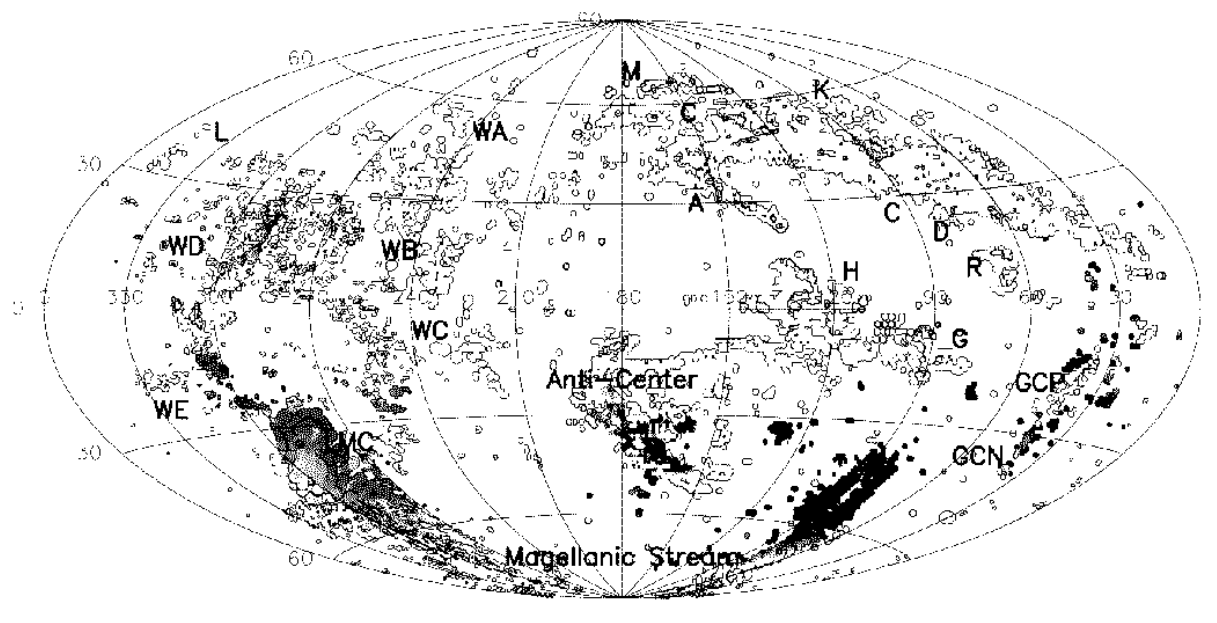

Figure 1: Aitoff projection all-sky map of the Galactic HVCs, in galactic coordinates, for $\mathrm{H}$ I column densities $>2 \times 10^{18} \mathrm{~cm}^{-2}$, based on the $21 \mathrm{~cm}$ data from Hulsbosch \& Wakker (1988) and Morras et al. (2000). Individual HVC complexes are indicated. Figure taken from Wakker (2004) [PLEASE CONTACT THE AUTHOR FOR A HIGH-QUALITY VERSION OF THIS FIGURE.]

about the chemical composition and spatial distribution of IVCs and HVCs. These studies have lead to a much improved understanding of the nature of these clouds (see also Wakker \& Richter 2004). In the following subsections I want to highlight some of these measurements.

\subsection{Distribution of Neutral Gas in the Halo}

The distribution of neutral gas in the halo of the Milky Way in the form of IVCs and HVCs can be studied best in the $\mathrm{HI} 21 \mathrm{~cm}$ line of neutral hydrogen. Fig. 1 shows the H I HVC sky (Aitoff projection) for H I column densities $>2 \times 10^{18} \mathrm{~cm}^{-2}$ based on the the $21 \mathrm{~cm}$ data from Hulsbosch \& Wakker (1988) and Morras et al. (2000). For $N(\mathrm{H} \mathrm{I})>2 \times 10^{18} \mathrm{~cm}^{-2}$ the sky-covering fraction of HVC H I gas is 30 percent (see, e.g., Murphy et al. 1995; Wakker 2004), while for $N(\mathrm{H} \mathrm{I})>7 \times 10^{17} \mathrm{~cm}^{-2}$ the covering fraction increases to almost 50 percent. Note that neutral gas structures with even lower H I column densities do exist. Such clouds lie below the detection limit of current $21 \mathrm{~cm}$ radio observations, but they can be observed using optical and FUV absorption line measurements (e.g., Richter et al. 2005a; see also Section 4.3). The H I HVC sky is divided into individual HVC "complexes" (for a list of names see Wakker 2001), as indicated in Fig. 1. The most prominent of these HVC complexes are complex A $(l \sim 150, b \sim+35)$, complex $\mathrm{C}(l \sim 30-150, b \sim+40)$, complex 
$\mathrm{H}(l \sim 130, b \sim 0)$, the Anti-Center Cloud $(l \sim 180, b \sim-30)$, and the Magellanic Stream $(l \sim 90-300, b \sim-30$ to -90$)$. Recently, Kalberla et al. (2005) have combined and newly reduced $21 \mathrm{~cm}$ data from the Leiden-Dwingeloo Survey (LDS; Hartmann \& Burton 1997) and the Villa-Elisa Survey (Morras et al. 2000) and have created a $\mathrm{H}$ I $21 \mathrm{~cm}$ all-sky survey (the Leiden-ArgentinaBonn survey; LAB survey) that represents an excellent data base to study the distribution of the HVC $\mathrm{H}$ I gas in the Milky Way halo.

Next to the large HVC complexes listed above there is a population of isolated, relatively small HVCs, commonly referred to as compact HVCs (CHVCs; Braun \& Burton 1999). These are sharply bounded in angular extent (angular sizes are $<2^{\circ}$, typically) and appear to have no kinematic or spatial connection to other HVC features. It was suggested that CHVCs are candidates for dark-matter halos filled with gas at megaparsec distances that are distributed throughout the Local Group of galaxies (Braun \& Burton 2000). However, recent distance estimates for these objects (Westmeier et al. 2005a) speak against this scenario.

Also the intermediate-velocity clouds in the halo cover a significant portion of the sky. Fig. 2 shows the H I IVC sky in the velocity range between -35 and $-90 \mathrm{~km} \mathrm{~s}^{-1}$ and for $\mathrm{H} \mathrm{I}$ column densities $>10^{19} \mathrm{~cm}^{-2}$, based on data of the H I Leiden-Dwingeloo Survey. Prominent IVC structures include the Intermediate-Velocity Arch (IV Arch; $l \sim 180, b \sim+70$ ), the Low-Latitude Intermediate-Velocity Arch (LLIV Arch; $l \sim 160, b \sim+30$ ), the IntermediateVelocity Spur (IV Spur; $l \sim 250, b \sim+60$ ), and the Pegasus-Pisces Arch (PP Arch; $l \sim 110, b \sim-50)$. From these data it follows that IVC $\mathrm{H}$ I gas with $N(\mathrm{H} \mathrm{I})>10^{19} \mathrm{~cm}^{-2}$ fills $\sim 40$ percent of the sky.

A more detailed review of the distribution of HVCs and IVCs in the Milky Way halo is provided by Wakker (2004).

\subsection{Distances to IVCs and HVCs}

Measuring the distance to IVCs and HVCs is of great importance to understand the nature and origin of these clouds, as the distance information together with the sky distribution (see previous section) provides a threedimensional view of these clouds around the Galaxy. Unfortunately, very little information about IVC and HVC distances is available. The reason for this is that reliable distance measurements of IVCs and HVCs are very difficult and achievable only for a small number of these clouds.

The most reliable (and only direct) method to measure IVC/HVC distances is the absorption-line method, in which one uses (mostly optical) highresolution spectra of stars with known distances in the direction of a halo cloud. If a halo star is located behind an IVC or HVC that has a sufficiently large gas-column density, one expects to see IVC/HVC absorption (e.g., from the optical Ca II lines) in the stellar spectrum. If the IVC/HVC is behind the star, no such absorption can occur. One thus can bracket the distance of the IVCs and HVCs within a range defined by the distances of the available background stars. This method has been used for a few IVCs and HVCs. 


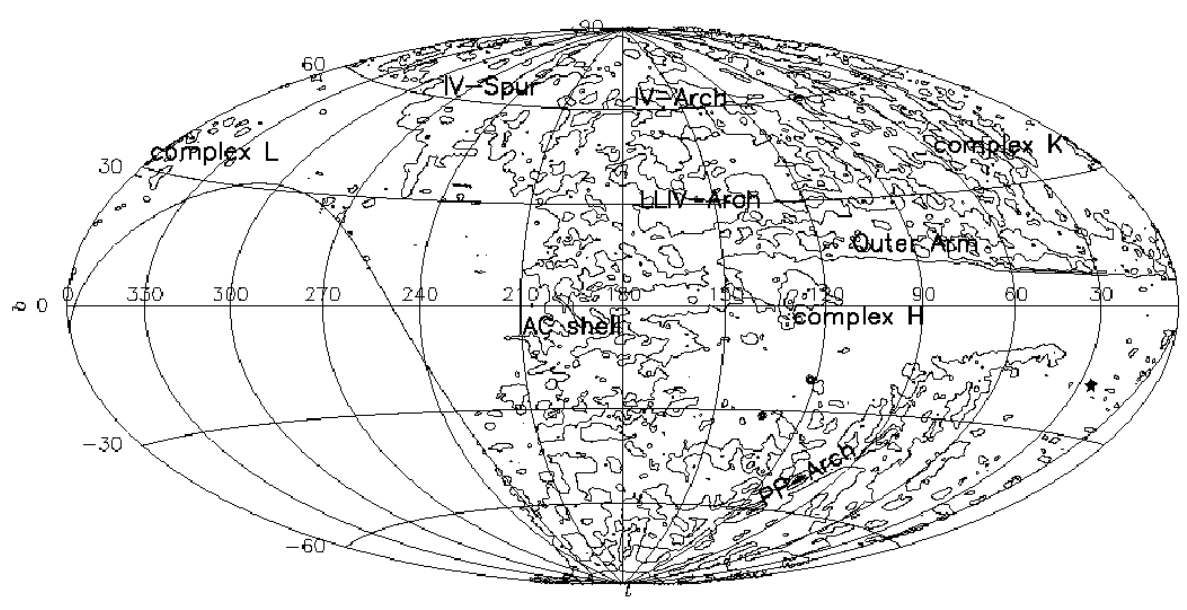

Figure 2: Aitoff projection all-sky map of the Galactic IVCs, in galactic coordinates, for H I column densities $>10^{19} \mathrm{~cm}^{-2}$ and velocities between -35 and $-90 \mathrm{~km} \mathrm{~s}^{-1}$, based on the $21 \mathrm{~cm}$ data from the Leiden-Dwingeloo Survey (LDS; Hartmann \& Burton 1997). Individual IVC complexes are indicated. Figure taken from Wakker (2004). [PLEASE CONTACT THE AUTHOR FOR A HIGH-QUALITY VERSION OF THIS FIGURE.]

IVCs with known distances are the IV Arch and the LLIV Arch (both have $d=0.5-2.0 \mathrm{kpc}$ ); for some other IVCs, upper distance limits of a few kpc have been derived (see Wakker 2001 and references therein). The few HVCs for which useful distance information is available are comlex A $(d=4-10$ kpc; van Woerden et al. 1999; Wakker 2001), complexes C and $\mathrm{H}(d>5 \mathrm{kpc}$; Wakker 2001), and complexes WE ( $d<13 \mathrm{kpc}$; Sembach et al. 1991) and WB $(d<16 \mathrm{kpc}$; Thom et al. 2005). These values suggest that most IVCs represent objects that are relatively nearby with typical distances of $<2 \mathrm{kpc}$, while many of the HVCs are more distant halo clouds with typical distances $>5 \mathrm{kpc}$. However, more of these distance measurements are required to better understand the distribution of intermediate- and high-velocity gas surrounding the Milky Way. Recently, a very large number of blue-horizontal branch stars in the halo at high galactic latitudes and in a large distance range $(d=0-90 \mathrm{kpc})$ have been identified in various observational surveys (e.g., Brown et al. 2004; Sirko et al. 2004; Christlieb et al. 2004). These stars can be used as background sources for Ca II absorption line measurements in the direction of IVCs and HVCs, so that important new IVC/HVC distance information is expected to become available during the next few years.

An indirect method to constrain distances of IVCs and HVCs is to measure the intensity of $\mathrm{H} \alpha$ emission from these clouds. As recent observations demonstrate, several IVCs and HVCs shine bright in $\mathrm{H} \alpha$ emission (e.g., Weiner et al. 2001; Putman et al. 2003), implying that the neutral clouds are surrounded 
by ionized gaseous envelopes. If the Milky Way's ionizing radiation field is responsible for the existence of these ionized gas layers, the measured $\mathrm{H} \alpha$ intensity in IVCs and HVCs serves as a measure for the distance to the ionization source (i.e., the Galactic stellar disk). Weiner et al. and Putman et al. have measured $\mathrm{H} \alpha$ emission in a number of IVCs and HVCs and conclude that many of these clouds are within a radius of $\sim 40 \mathrm{kpc}$ from the Galaxy. This therefore favors a scenario in which most of the large IVCs and HVCs are located within the Milky Way halo but are not Local Group objects at megaparsec distances. A major problem with the $\mathrm{H} \alpha$ method is, however, that next to radiation from the Milky Way disk collisional processes also may contribute to the ionization of the gaseous envelopes of these clouds. This can be seen in the case of the Magellanic Stream, which most likely represents gaseous material torn out of the Magellanic Clouds during their tidal interaction with the Milky Way. The Magellanic Stream is expected to be at least $50 \mathrm{kpc}$ away and thus is too distant to be substantially ionized by the Galaxy's radiation field. Yet, the Stream shows $\mathrm{H} \alpha$ emission at a level of up to $\sim 400 \mathrm{mR}$ (Putman et al. 2003), suggesting that it has an ionized envelope that is produced by collisional processes as the Stream is ramming into the hot, extended Corona that surrounds the Milky Way (Sembach et al. 2003). Due to the unknown contribution of collisional processes to the ionization fraction in the envelopes of IVCs and HVCs, distance estimates from $\mathrm{H} \alpha$ fluxes are affected by large systematic uncertainties.

An indirect method to obtain information about the distances of the mysterious CHVCs in the Milky Way halo is to consider the $21 \mathrm{~cm}$ brightness temperature of extraplanar and circumgalactic $\mathrm{H}$ I structures in M31, the other large spiral galaxy in the Local Group that is $\sim 780 \mathrm{kpc}$ away (see also Section 4.1). Under the assumption that our sister galaxy also comprises a population of H I CHVCs with similar properties (i.e., radial distribution and emission characteristics mimic those of the Milky Way CHVCs), the comparison of measured $21 \mathrm{~cm}$ brightness temperatures of the Galactic CHVCs with limits derived for the M31 CHVC population at $780 \mathrm{kpc}$ distance provides an estimate for the distance of the CHVCs surrounding the Milky Way. Using $\mathrm{H}_{\mathrm{I}} 21 \mathrm{~cm}$ data from the Effelsberg $100 \mathrm{~m}$ radio telescope, Westmeier et al. (2005a) conclude from the measured limits for the M31 CHVC population that the Milky Way's CHVCs cannot have distances larger than $\sim 60 \mathrm{kpc}$. These measurements (just like the $\mathrm{H} \alpha$ observations discussed above) therefore suggest that the Galactic H I HVCs (including the CHVCs) are relatively nearby and thus represent a circumgalactic rather than an intergalactic/Local Group phenomenon.

\subsection{Metal Abundances and Origin of IVCs and HVCs}

Apart from the distance, the most valuable information about the origin of IVCs and HVCs comes from studies of the chemical composition of these clouds. Ultraviolet absorption-line spectroscopy is the most sensitive and accurate method to measure metal abundances and physical properties in 


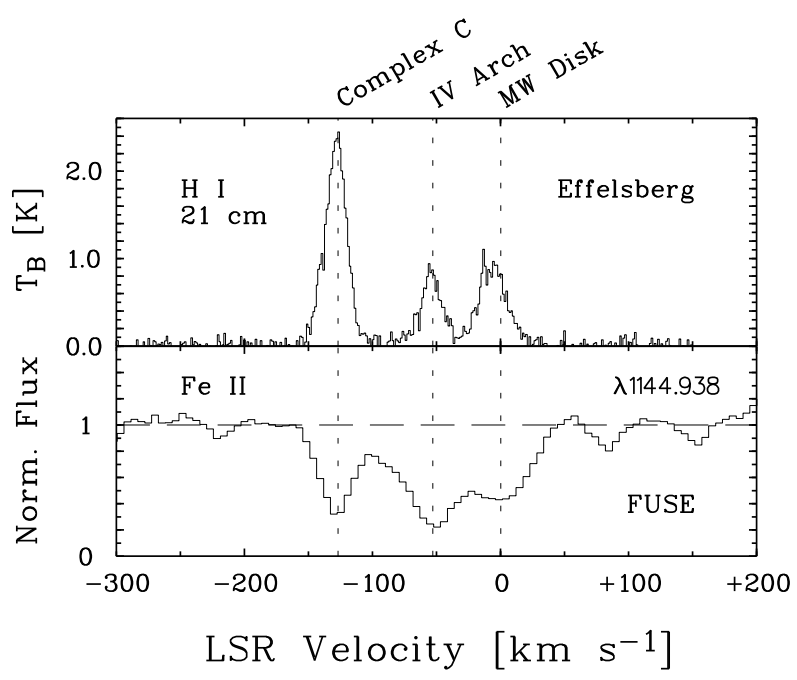

Figure 3: Velocity profiles of the H I $21 \mathrm{~cm}$ emission and Fe II $\lambda 1144.9 \mathrm{FUV}$ absorption in the direction of the quasar PG $1259+593(l=120.6, b=+58.1)$. Next to the local emission/absorption from Galactic disk gas near zero velocities, IVC and HVC emission/absorption is seen at $-55 \mathrm{~km} \mathrm{~s}^{-1}$ and -130 $\mathrm{km} \mathrm{s}^{-1}$, indicating neutral halo gas associated with the IV Arch and HVC complex C. Figure adapted from Richter et al. (2001b).

IVCs and HVCs, where typical gas densities are significantly lower than in Galactic disk clouds. The FUSE satellite has sufficient sensitivity and spectral resolution to investigate absorption at $\lambda \leq 1187 \AA$ in the Galactic halo and beyond along a large number of sight lines. At longer wavelengths $(\lambda>$ $1150 \AA$ ), the STIS instrument installed on the Hubble Space Telescope (HST) provides additional information about a number of atomic species and the Ly $\alpha$ absorption line of neutral hydrogen near $1215.7 \AA$. Combining data from these instruments thus provides a particularly powerful tool to investigate metal abundances in intermediate- and high-velocity clouds.

During the last decade, a large number of measurements with FUSE, STIS, and other instruments have provided important new results on the chemical composition of the Galactic IVCs and HVCs (e.g., Richter et al. 1999; Wakker et al. 1999; Richter et al. 2001a; Richter et al. 2001b; Collins et al. 2003; Tripp et al. 2003; Sembach et al. 2004a). These studies reveal disparate chemical compositions for several IVCs and HVCs in different directions in the sky, indicating that many of these clouds cannot have a common origin. Overall metal abundances in IVCs and HVCs typically vary between $\sim 0.1$ and $\sim 1.0$ solar. In general, the chemical make-up of most of the IVCs and some of the HVCs can be explained successfully by the galactic fountain model (Shapiro \& Field 1976; Houck \& Bregman 1990), in which gas is ejected out of the Galactic disk by supernova explosions and is falling back onto the disk in 
the form of neutral gas clouds. Gas that is participating in this circulatory pattern should have a nearly solar metallicity reflecting that of their place of origin (i.e., the Milky Way disk). Prominent examples for such solarmetallicity galactic fountain clouds are the Intermediate-Velocity Arch (IV Arch; Richter et al. 2001b), the Low- Latitude Intermediate Velocity Arch (LLIV Arch; Richter et al. 2001a), and HVC complex M (Wakker 2001). There are, however, a number of HVCs whose abundances are inconsistent with the Galactic fountain model. One such case is the Magellanic Stream, which has abundances close to those of the Small Magellanic Cloud (SMC; $\sim 0.3$ solar). The Stream is believed to be tidally stripped out of the SMC system during the last encounter with the Galaxy (e.g., Wannier \& Wrixon 1972; Lu et al. 1998; Sembach et al.2001). High-velocity cloud complex C has an even lower abundance $(\sim 0.1-0.3$ solar; Wakker et al. 1999; Richter et al. 2001b; Tripp et al. 2003) that is inconsistent with gas originating in the disk of the Galaxy or in the Magellanic Clouds. Thus, complex C might represent metalpoor material accreted from the intergalactic medium. Possibly, mass shed by metal-poor halo red giants contributes to the infall of low-metallicity gas, too (de Boer 2004). Accretion of substantial quantities of metal-poor gas in the form of HVCs would have a significant influence on the chemical evolution of the Milky Way.

As an example for recent metal abundance measurements in Galactic IVCs and HVCs we discuss the abundance pattern in IVC/HVC gas in the direction of the quasar PG $1259+593(l=120.6, b=+58.1)$. This sightline is particularly well suited to explore the metal content of IVC/HVC gas with FUV absorption spectroscopy, as it passes two different Galactic halo clouds (one IVC and one HVC) that have different radial velocities. The FUV spectrum of PG $1259+593$ therefore allows us to directly compare the chemical abundances in two clouds using the same spectral data. In addition, PG $1259+593$ lies in a direction in the sky where the local Galactic foreground gas in the disk has a very low neutral gas column density. This is an advantage, since the many absorption lines from molecular hydrogen $\left(\mathrm{H}_{2}\right)$ in the disk gas are weak in this direction and thus $\mathrm{H}_{2}$ line blending with IVC and $\mathrm{HVC}$ components is much less severe than along other QSO sightlines. Richter et al. (2001b) have analyzed interstellar ultraviolet absorption lines in $\mathrm{HVC}$ complex $\mathrm{C}$ and the IV Arch in the direction of the quasar PG 1259+593 using FUSE and STIS data. Fig. 3 shows the velocity structure of interstellar gas in the direction of PG $1259+593$. In the upper panel, the velocity profile of the $\mathrm{HI} 21 \mathrm{~cm}$ emission (based on observations with the Effelsberg $100 \mathrm{~m}$ radio telescope) is plotted against the LSR velocity. Next to the local $\mathrm{H}$ I $21 \mathrm{~cm}$ emission from Galactic disk gas near zero velocities, IVC and HVC H I emission is seen at $-55 \mathrm{~km} \mathrm{~s}^{-1}$ and $-130 \mathrm{~km} \mathrm{~s}^{-1}$, indicating halo gas related to the IV Arch and HVC complex C. In the lower panel of Fig. 3 we show the absorption pattern in the Fe II $\lambda 1144.938$ line, based on the FUSE observations of PG 1259+593. Fe II absorption by the IV Arch and complex C are clearly visible and (despite the lower resolution) the velocity pattern perfectly matches that of the $\mathrm{H}$ I $21 \mathrm{~cm}$ emission. Other atomic species detected (in absorption) in the IV 


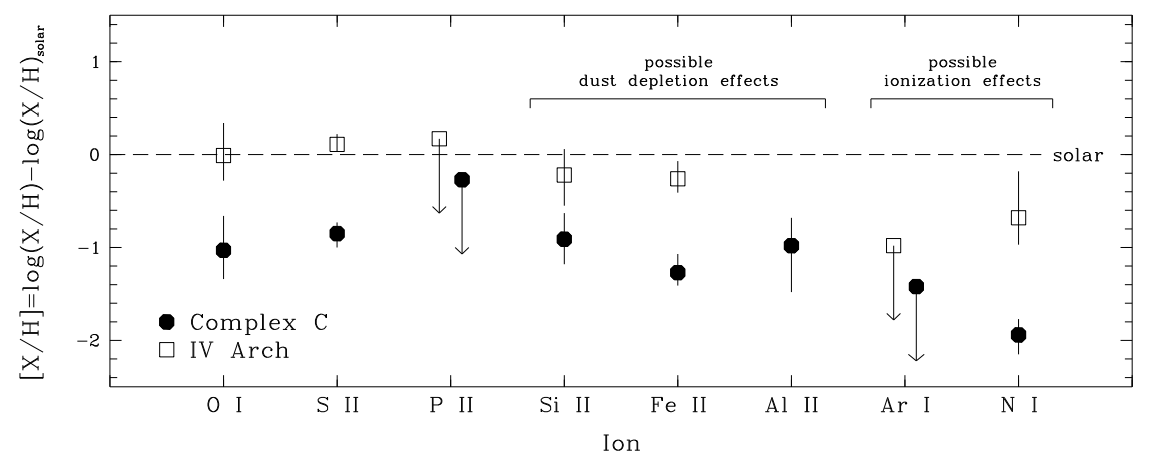

Figure 4: Normalized interstellar gas-phase abundances in complex $\mathrm{C}$ and the IV Arch. Abundances in complex $\mathrm{C}$ are systematically lower than in the IV Arch, suggesting a different enrichment history of both clouds. Figure taken from Richter et al. (2001a).

Arch and complex C include D i, C II, N I, N II, O I, Al II, Si II, P II, S II, Ar I, and Fe III (Richter et al. 2001b; Sembach et al. 2004a).

The $\mathrm{O}$ I/H I ratio provides the best measure of the overall metallicity in the diffuse interstellar medium, because ionization effects do not alter the ratio, and oxygen is at most only lightly depleted from the gas into dust grains. For complex C, Richter et al. find an oxygen abundance of $0.09_{-0.05}^{+0.13}$ solar, consistent with the idea that complex $\mathrm{C}$ represents the infall of low-metallicity gas onto the Milky Way. In contrast, the oxygen abundance in the IV Arch is 0.98 ${ }_{-0.46}^{+1.21}$ solar, which points to a Galactic origin. Similar abundance differences between the IV Arch and complex $\mathrm{C}$ are also found in other elements, but here, dust depletion and ionization effects have to be taken into account for the interpretation of the observed abundance ratios. The abundance pattern for various elements such as oxygen, sulfur, phosphorus, silicon, iron, aluminum, argon, and nitrogen in the IV Arch and complex $\mathrm{C}$ is shown in Fig. 4. The abundance measurements toward PG $1259+593$ demonstrate that various different processes are responsible for the phenomenon of intermediatehigh-velocity neutral gas clouds in the halo of the Milky Way - they cannot have a single origin.

An extensive summary of absorption line measurements in Galactic halo clouds is provided by Wakker (2001).

\subsection{Small-Scale Structure in IVCs and HVCs}

The gaseous halo of the Milky Way is an extreme multi-phase medium with temperatures ranging from 50 to several million Kelvin. Although the large IVC and HVC complexes like the IV Arch, complex $\mathrm{C}$ and the Magellanic Stream may span a depth of several kpc in the halo, small-scale structure in this gas is present down to scales of several AU (e.g., Meyer \& Lauroesch 1999). 


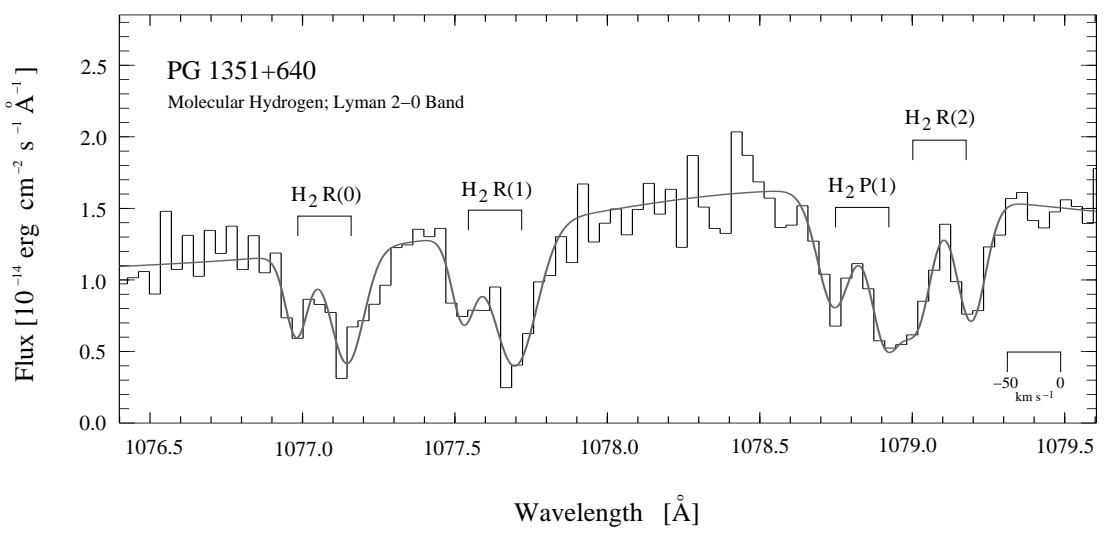

Figure 5: Excerpt of the FUSE spectrum of PG $1351+640$ in the wavelength range between 1076.5 and $1079.5 \AA$, sampling the $\mathrm{H}_{2} 2-0$ Lyman band. The individual $\mathrm{H}_{2}$ lines present in the data are labeled above the spectrum. $\mathrm{H}_{2}$ absorption from the rotational levels $J=0,1$ and 2 is seen in two components at 0 and $-50 \mathrm{~km} \mathrm{~s}^{-1}$, representing $\mathrm{H}_{2}$ gas from the local Milky Way and the IV Arch (core IV 19), respectively. The solid gray line shows a two-component Gaussian fit of the spectrum that nicely reproduces the absorption pattern seen in the FUSE data. Figure taken from Richter et al. (2003a).

Studying this small-scale component in Galactic halo clouds not only provides an insight into the internal structure of IVCs and HVCs, but also yields important information about physical processes in the interstellar medium in general.

Molecular hydrogen is an excellent diagnostic tool to investigate smallscale structure and physical conditions in IVCs and HVCs. A large number of $\mathrm{H}_{2}$ absorption lines from the Lyman and Werner band is present in the FUV range between 900 and $1200 \AA$. The $\mathrm{H}_{2}$ abundance in the diffuse ISM is determined by a balance of the formation of molecules on the surface of dust grains and the $\mathrm{H}_{2}$ destruction by the dissociating UV radiation (see Richter et al. 2003a). For known photoabsorption and grain formation rates one can estimate the hydrogen volume density from the measured $\mathrm{HI}$ and $\mathrm{H}_{2}$ column densities. The size of an $\mathrm{H}_{2}$ absorbing structure, $D$, then can easily be calculated from $N(\mathrm{H} \mathrm{I})$ and $n_{\mathrm{H}}$. Also the rotational excitation of the $\mathrm{H}_{2}$ molecules can be used to investigate physical conditions in the gas. The lowest rotational energy states of $\mathrm{H}_{2}$ (rotational levels $J=0$ and 1 ) are usually excited by collisions, so that the column density ratio $N(1) / N(0)$ serves as a measure for the kinetic temperature of the gas. In addition, the population of the higher rotational states (determined by UV photon pumping) indicates the strength of the local UV radiation field.

A number of positive detections of $\mathrm{H}_{2}$ absorption with FUSE and ORFEUS has been reported for both IVCs and HVCs (e.g., Richter et al. 1999; 
Gringel et al. 2000; Bluhm et al. 2001; Richter et al. 2001c; Sembach et al. 2001; Richter et al. 2003a). Positive detections include the IV Arch, LLIV Arch, IV Spur, complex gp, the Draco cloud, LMC-IVC, LMC-HVC, and the Magellanic Stream (see also Richter \& de Boer 2004). In all cases, the observed column densities are low $\left(\log N\left(\mathrm{H}_{2}\right) \leq 17\right)$, implying that the $\mathrm{H}_{2}$ resides in a predominantly neutral gas phase. As expected, $\mathrm{H}_{2}$ absorption mostly occurs in halo clouds that have a high metal and dust abundance, thus preferably in IVCs rather than in HVCs (e.g., Richter et al.1999). As an example for $\mathrm{H}_{2}$ absorption in a Galactic IVC we show in Fig. 5 the FUSE spectrum of the quasar PG $1351+640$ in the range between 1076.5 and $1079.5 \AA$, where a number of $\mathrm{H}_{2}$ lines from various rotational states are present. Halo $\mathrm{H}_{2}$ absorption at negative intermediate velocities from gas in the IV Arch (core IV 19) is clearly visible in the various $\mathrm{H}_{2}$ lines shown.

With FUSE, Richter et al. (2003a) have systematically studied the properties of the $\mathrm{H}_{2}$ gas in IVCs towards a large number (56) of mostly extragalactic background sources. The sample includes 61 IVC components with $\mathrm{H}$ I column densities $\geq 10^{19} \mathrm{~cm}^{-2}$ and radial velocities $25 \leq\left|v_{\mathrm{LSR}}\right| \leq 100 \mathrm{~km} \mathrm{~s}^{-1}$. In FUSE spectra with good signal-to-noise ratios $(\mathrm{S} / \mathrm{N}>8$ per resolution element) they find 14 clear detections of $\mathrm{H}_{2}$ in IVC gas with $\mathrm{H}_{2}$ column densities between $10^{14}$ and $10^{17} \mathrm{~cm}^{-2}$. In lower $\mathrm{S} / \mathrm{N}$ data, $\mathrm{H}_{2}$ absorption in IVC gas is tentatively detected in an additional 17 cases. The molecular hydrogen fraction in these clouds, $f=2 N\left(\mathrm{H}_{2}\right) /\left[N(\mathrm{H} \mathrm{I})+2 N\left(\mathrm{H}_{2}\right)\right]$, varies between $10^{-6}$ and $10^{-3}$. This suggests that the $\mathrm{H}_{2}$ lives in a relatively dense, mostly neutral gas phase that probably is linked to the cold neutral medium (CNM) in these clouds. Under the assumption of a $\mathrm{H}_{2}$ formation-dissociation equilibrium one can determine the hydrogen volume density and the thickness of the absorbing structure. The $\mathrm{H}_{2}$ photoabsorption rate in the halo depends on the mean ultraviolet radiation field at a height $z$ above the Galactic plane. The models of Wolfire et al. (1995) predict that that the radiation field and the $\mathrm{H}_{2}$ photoabsorption rate at $\sim 1 \mathrm{kpc}$ above the disk is approximately 50 percent of that within the disk. If one assumes that the $\mathrm{H}_{2}$ grain formation rate in IVCs is roughly similar to that within the disk, the $\mathrm{H}_{2}$ and $\mathrm{H}$ I column densities measured for the Richter et al.IVC sample imply mean hydrogen volume densities of $n_{\mathrm{H}} \approx 30 \mathrm{~cm}^{-3}$ and linear diameters of the $\mathrm{H}_{2}$ absorbing structures of $D \approx 0.1 \mathrm{pc}$. Moreover, if one considers the rotational excitation of the halo $\mathrm{H}_{2}$ gas that can be measured for some of the IVC sightlines, one finds for the kinetic temperature of this gas a conservative upper limit of $T_{\text {kin }} \leq 300 \mathrm{~K}$. Given the relatively high detection rate of $\mathrm{H}_{2}$ in these clouds, the measurements indicate that the CNM phase in IVCs is ubiquitous and therefore represents a gas phase that is characteristic for the denser, neutral regions in the halo. Most likely, the CNM filaments are embedded in a more tenuous gas phase that corresponds to the warm neutral medium (WNM).

The detection of $\mathrm{H}_{2}$ absorption in a low-column density IVC towards the LMC star Sk -6880 (Richter et al.2003b) demonstrates that even smaller filaments at $\mathrm{AU}$ scales exist in the halo. The observed gas clumps must have very high hydrogen volume densities (almost $10^{3} \mathrm{~cm}^{-3}$ ) and relatively low gas 
temperatures $(T<50 \mathrm{~K})$. They probably are related to the so-called tiny-scale atomic structures (TSAS), small-scale structures that also have been found in the disk of the Milky Way (Heiles et al.1997; Heithausen 2004). Many aspects that concern the physical nature of these tiny filaments (e.g., formation processes, thermal pressures, dust content, etc.) are not well understood, and more data are required to explore these intriguing objects in more detail.

\section{The Galactic Corona}

\subsection{Overview}

That the disk of our Milky Way is surrounded by an envelope of hot gas was first proposed by Spitzer (1956). Spitzer considered the presence of such a "Galactic Corona" (according to the solar corona) as necessary to explain spectroscopic observations that have been made some years earlier by Münch (1952) and Münch \& Zirin (1961). They had found interstellar Ca II absorption from neutral gas clouds spread over a large velocity range $\left(\sim 50 \mathrm{~km} \mathrm{~s}^{-1}\right)$ towards $\mathrm{O}$ and $\mathrm{B}$ stars at high galactic latitudes and large distances $(>500$ pc) from the Galactic plane. This was the first detection of the intermediateand high-velocity clouds in absorption. Due to the low neutral gas density so far above the Galactic plane, Spitzer argued that these clouds should not exist for long, but disperse at time scales of $\sim 10^{7}$ years, unless they would be embedded in a thin but hot, highly ionized gaseous medium that would provide the necessary thermal pressure to confine these clouds. While the presence of the neutral high-velocity clouds was confirmed shortly after by $\mathrm{H} \mathrm{I}$ $21 \mathrm{~cm}$ radio observations (Muller et al. 1963; see Section 2.2), it took about 20 years to find compelling observational evidence for the hot coronal gas in which the IVCs and HVCs are embedded.

In the mid-seventies, observations with the Copernicus satellite (e.g., Jenkins et al. 1974) discovered the widespread presence of five-times ionized oxygen (O vI) within the disk of the Milky Way. In addition, diffuse X-ray emission was found toward high latitudes in rocket experiments (Williamson et al. 1974). To account for this newly detected ubiquitous hot interstellar gas phase, and also for the high-velocity clouds that are falling towards the Galactic disk, Shapiro \& Field (1976) developed the model of the galactic fountain, in which the hot gas is produced by supernova explosions in the disk of the Milky Way. This gas forms cavities, which expand (due to overpressure) and eventually break out of the disk. Hot gas then would stream into the Milky Way halo several kiloparsecs above the disk, forming a hot, gaseous corona of the Milky Way, as proposed by Spitzer twenty years earlier. Part of this gas would be able to cool, forming some of the intermediate- and highvelocity clouds. In the same way as for the neutral halo clouds, absorption line spectroscopy in the UV and FUV is well suited to study hot gas in the circumgalactic environment of the Milky Way, as the UV and FUV range contains a number of lines from highly-ionized species, such as C IV, N v, and 
$\mathrm{O}$ vi. These lines sample gas in the temperature range between $1 \times 10^{5}$ and $5 \times 10^{5} \mathrm{~K}$ and thus provide important information about the distribution and physical properties of hot gas in the halo of the Milky Way (see also de Boer 2004).

\subsection{Distribution of $\mathrm{O}$ VI in the Halo}

The first absorption-line studies of the Galactic Corona were based on observations of Si IV, C IV, and N v with the International Ultraviolet Explorer (IUE) and the HST (Savage \& de Boer 1979, 1981; Sembach \& Savage 1992; Savage et al.1997). The best possible way to study the hot halo component of the Milky Way is absorption spectroscopy of $\mathrm{O}$ VI, which has as an ionization potential as large as $\sim 114 \mathrm{eV}$. O VI traces either collisionally ionized, hot gas at temperatures near $3 \times 10^{5} \mathrm{~K}$, or low density gas that is exposed to a very intense UV radiation field, or a mixture of both. O VI does not trace the very hot gas phase (with temperatures exceeding $10^{6} \mathrm{~K}$ ) that likely exists in the coronal gas and that is responsible for the soft X-ray emission. Gas at temperatures around $3 \times 10^{5} \mathrm{~K}$ cools rapidly and $\mathrm{O}$ VI absorption therefore is expected to trace the interface regions between the hot $\left(10^{6} \mathrm{~K}\right)$ and the warm $\left(10^{4} \mathrm{~K}\right)$ gas phase, e.g., in cooling flows, cooling bubbles, and mixing layers.

A rather simple model to describe the density distribution of the hot gas as a function of the vertical distance from the Galactic plane ( $z$ height) is to assume an exponential stratification. With $n_{0}$ as the mid-plane gas volume density and $h$ as the scale-height the gas-density at a given $z$ can be expressed as $n(z)=n_{0} \exp (-|z| / h)$. High-ion absorption line measurements in sightlines through the halo provide a direct estimate of the stratification of the hot gas away from the Galactic plane. For a given sightline in the direction $(l, b)$, the column density for the ion $\mathrm{X}, N(\mathrm{X})$, is measured up to a height $|z|$ above the disk, at which the background source is located (these background sources may be halo stars or extragalactic objects). Comparing $N(\mathrm{X}) \sin |b|$ with $|z|$ for a large number of sightlines, one can fit an exponential distribution (as shown above) to the data points and derive the scale height $h(\mathrm{X})$. Such a $N(\mathrm{X}) \sin |b|$ vs. $|z|$ analysis has been done by Savage et al. (1997) for the species Si IV, C IV, and Nv. Using spectra from the Goddard High Resolution Spectrograph (GHRS) on HST they derive exponential scale heights of $h(\mathrm{Si} \mathrm{IV})=5.1 \pm 0.7$ $\mathrm{kpc}, h(\mathrm{C} \mathrm{IV})=4.4 \pm 0.6 \mathrm{kpc}$, and $h(\mathrm{Nv})=3.9 \pm 1.4 \mathrm{kpc}$. In contrast, for the sample of objects the scale height derived for neutral hydrogen is just $h(\mathrm{HI})=0.30 \pm 0.03 \mathrm{kpc}$, showing that the neutral gas phase is (with the exception of the IVCs and HVCs) concentrated in a thin layer in the Galactic disk. Another possibility to estimate the scale height of the hot gas in the halo is to analyze individual absorption profiles of high ions assuming that the gas is co-rotating with the underlying disk. Savage et al. (1997) show that for C IV such an analysis results in a scale height of $\sim 4.5 \mathrm{kpc}$, thus very similar to the one derived with the $N(\mathrm{X}) \sin |b|$ vs. $|z|$ method.

Using ORFEUS data, Widmann et al. (1998) have presented the first systematic study of O VI absorption in the halo. With the availability of a large 
number of FUSE absorption spectra from extragalactic background sources in 1999 our knowledge about the $\sim 3 \times 10^{5} \mathrm{~K}$ gas component in the halo (as traced by O VI absorption) has improved substantially. Wakker et al. (2003), Savage et al. (2003), and Sembach et al. (2003) present a large survey of O vi absorption along 102 lines of sight through the Milky Way halo. They find strong $\mathrm{O}$ VI absorption in a radial-velocity range from approximately -100 to $+100 \mathrm{~km} \mathrm{~s}^{-1}$ with logarithmic O vi column densities (in units $\mathrm{cm}^{-2}$ ) ranging from 13.85 to 14.78 (Savage et al. 2003). At these radial velocities, the O VI absorbing gas should be located in the thick disk and/or halo of the Milky Way. The distribution of the O VI absorbing gas in the thick disk and halo is not uniform, but appears to be quite irregular and patchy. A simple model assuming a symmetrical plane-parallel patchy layer of $\mathrm{O}$ VI absorbing material provides a rough estimate for the exponential O vi scale height in the halo. Savage et al. (2003) find $h(\mathrm{O} v \mathrm{vI}) \sim 2.3 \mathrm{kpc}$ with an $\sim 0.25$ dex excess of $\mathrm{O}$ vI in the northern Galactic polar region. The correlation of O VI with other ISM tracers, such as soft X-ray emission, $\mathrm{H} \alpha$, and $\mathrm{H}$ I $21 \mathrm{~cm}$, is rather poor (Savage et al. 2003). Mixing of warm and hot gas and radiative cooling of outflowing hot gas from supernova explosions in the disk could explain the irregular distribution of $\mathrm{O}$ vi absorbing gas in the halo of the Milky Way.

\subsection{Highly-Ionized High-Velocity Clouds}

O VI absorption towards extragalactic background sources is observed not only at radial velocities $\leq 100 \mathrm{~km} \mathrm{~s}^{-1}$, but also at higher velocities (Wakker et al. 2003; Sembach et al. 2003). These detections imply that next to the Milky Way's hot "atmosphere" (i.e., the Galactic Corona) individual pockets of hot gas exist that move with high velocities through in the circumgalactic environment of the Milky Way. Such high-velocity O VI absorbers may contain a substantial fraction of the baryonic matter in the Local Group in the form of ionized hydrogen (e.g., Cen \& Ostriker 1999).

From their FUSE survey of high-velocity O vi absorption Sembach et al. (2003) find that probably more than 60 percent of the sky at high velocities is covered by ionized hydrogen (associated with the O VI absorbing gas) above a column density level of $\log N\left(\mathrm{H}^{+}\right)=18$, assuming a metallicity of the gas of 0.2 solar. Some of the high-velocity O VI detected with FUSE appears to be associated with known high-velocity $\mathrm{H}$ I $21 \mathrm{~cm}$ structures (e.g., the high-velocity clouds complex A, complex C, the Magellanic Stream, and the Outer Arm). Other high-velocity O vi features, however, have no counterparts in $\mathrm{H} \mathrm{I} 21 \mathrm{~cm}$ emission. The high radial velocities for most of these $\mathrm{O}$ VI absorbers are incompatible with those expected for the hot coronal gas (even if the coronal gas motion is decoupled from the underlying rotating disk). A transformation from the Local Standard of Rest to the Galactic Standard of Rest and the Local Group Standard of Rest velocity reference frames reduces the dispersion around the mean of the high-velocity O vi centroids (Sembach et al.2003; Nicastro et al. 2003). This can be interpreted as evidence that some of the $\mathrm{O}$ VI high-velocity absorbers are intergalactic clouds in the Local 
Group rather than clouds directly associated with the Milky Way. However, it is extremely difficult to discriminate between a Local Group explanation and a distant Galactic explanation for these absorbers. The presence of intergalactic O VI absorbing gas in the Local Group is in line with theoretical models that predict that there should be a large reservoir of hot gas left over from the formation of the Local Group (see, e.g., Cen \& Ostriker 1999). However, further FUV absorption line measurements and additional X-ray observations will be required to test this interesting idea.

It is unlikely that the high-velocity O VI is produced by photoionization. Probably, the gas is collisionaly ionized at temperatures of several $10^{5} \mathrm{~K}$. The $\mathrm{O}$ VI then may be produced in the turbulent interface regions between very hot $\left(T>10^{6} \mathrm{~K}\right)$ gas in an extended Galactic Corona and the cooler gas clouds that are moving through this hot medium (see Sembach et al. 2003). Evidence for the existence of such interfaces also comes from the comparison of absorption lines from neutral species like $\mathrm{O}$ I with absorption from highly-ionized species like O vi (Fox et al. 2004).

\section{Circumgalactic Gas in other Galaxies}

\subsection{Extraplanar $\mathrm{H}_{\text {I }}$ Gas in Galaxies}

The measurements of the Galactic IVCs and HVCs suggest that circumgalactic neutral gas clouds play a fundamental role in the evolution of the Milky Way. One thus would expect that also other galaxies consist of such circumgalactic gaseous components, and that HVCs represent a common phenomenon for spiral galaxies in the local Universe. Finding HVCs in the halos of other galaxies is challenging, however, due to the ambitious observational requirements, such as good mass sensitivity, a large field-of-view, and high spatial resolution. In recent years, great progress has been made to identify and measure extraplanar neutral gas structures in other nearby galaxies. Positive detections of discrete extraplanar $\mathrm{H}$ I clouds have been reported for the galaxies M31, M51, M81, M101, NGC 891, and many others (see Oosterloo 2004 for a review and references therein). Extensive H I halos are found in several other cases. These structures extend more than $10 \mathrm{kpc}$ away from the $\mathrm{H}$ I disk of the galaxies and appear to rotate slower than the underlying gaseous disk. Outflows as well as tidal interactions with surrounding satellite galaxies both are believed to contribute to the neutral gas flow in the halos of these galaxies.

A good example for HVCs in external galaxies is Andromeda (M31), next to the Milky Way the other large spiral galaxy in the Local Group. Using the Green Bank Telescope, Thilker et al.(2004) have found around twenty $\mathrm{H}$ I clouds in $21 \mathrm{~cm}$ emission in the halo of M31. They are located within $\sim 200 \mathrm{~km} \mathrm{~s}^{-1}$ of the systemic velocity of M31, thus in range similar to what is found for the Milky Way HVCs. Follow-up observations by Westmeier et al. (2005b) with the Westerbork Synthesis Radio Telescope have confirmed 


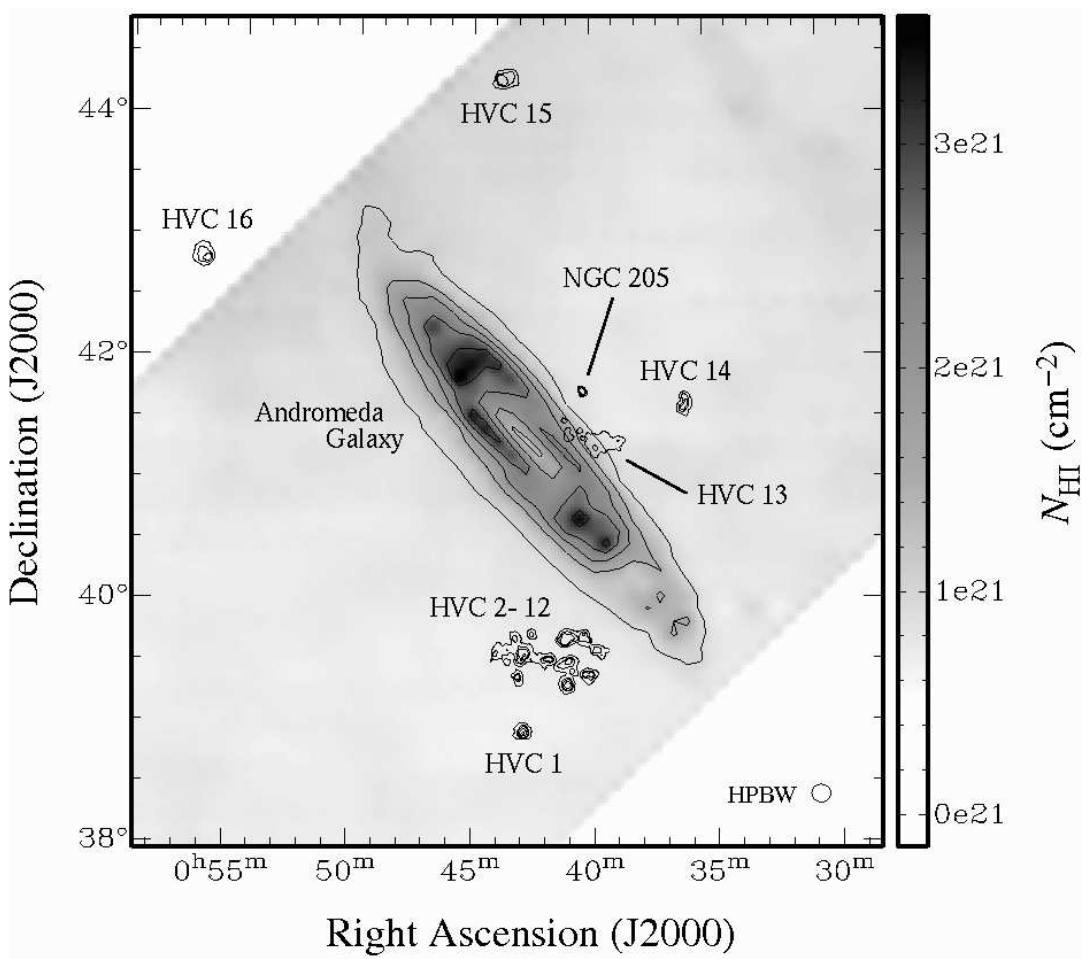

Figure 6: Distribution of H I gas clouds in the halo of M31 (figure adapted from Westmeier et al. 2005a, 2005b). Several gas complexes are evident at projected distances of several kpc above the disk of M31, demonstrating that the second large spiral galaxy in the Local Group also possesses a population of high-velocity clouds. HVCs and galaxies in the field are labeled accordingly.

the presence of the H I HVCs around M31. The derived H I column densities $\left(\sim 10^{19}-10^{20} \mathrm{~cm}^{-2}\right)$ and $\mathrm{H}$ I masses $\left(\sim 10^{4}-10^{6} M_{\odot}\right)$ are in line with values estimated for the Galactic HVC population. Also the M31 HVCs most likely are confined by a hot gaseous corona. Like the Milky Way, the Andromeda galaxy is surrounded by a number of satellite galaxies. Morphological and kinematical properties of the M31 HVCs therefore suggest that some of these clouds have a tidal origin, while other gaseous structures around M31 may represent fountain gas or material that is infalling from intergalactic space (see Westmeier et al. 2005b). Fig. 6 shows the population of HVCs around M31, based on the data by Westmeier et al. (2005a, 2005b).

\subsection{Diffuse Hot Gas around Galaxies}

$\mathrm{H} \alpha$ observations of edge-on spiral galaxies show the presence of vertically extended layers of diffuse ionized gas (DIG) above the disk. These measurements suggest that galactic-fountain processes (see Section 3) trigger the flow 
of gas from the disk into the halo and back, and that these fountain processes are common in spiral galaxies. Studies of DIG layers in the halos of galaxies at low redshift further indicate that their vertical extent and their brightness in the $\mathrm{H} \alpha$ emission depend on the level of the underlying star formation in the disk (e.g., Rand 1996; Rossa \& Dettmar 2003), implying that the bulk of the ionized gas is produced by supernova explosions. Therefore, ionized gas layers in the halos of disk galaxies trace the disk-halo flow of the metalenriched interstellar medium, while extraplanar H I structures seen in these systems predominantly represent the return flow of this gas (after substantial cooling) and the infall of material from outside. Spiral galaxies are also found to possess highly structured clouds of absorbing dust extending to several kpc distances from their mid-planes (Howk \& Savage 1997, 1999). The most impressive example is the multiphase halo of NGC 891, which exhibits numerous high- $z$ dust structures. Many of these structures contain $\sim 10^{5} M_{\odot}$ of gas and may be the sites of star formation (Howk \& Savage 2000).

In addition to these $\mathrm{H} \alpha$ and dust absorption observations, recent X-ray measurements of nearby edge-on galaxies with Chandra and XMM-Newton have shown that these systems consist of gigantic gaseous envelopes of hot, highly-ionized gas (e.g., Wang et al. 2001, 2005; Strickland et al. 2004). These coronae most likely resemble the extended hot halo of our own galaxy. Near the disks (i.e., at small vertical distances from the galactic plane), X-ray emission and $\mathrm{H} \alpha$ emission are strongly correlated. This supports the idea that both methods trace different temperature and density regimes of the same extraplanar ionized gas component. Also the X-ray luminosity of the coronal gas in disk galaxies is proportional to the star-formation rate in these systems (and to the total stellar mass). However, the observed X-ray luminosities can account only for a small fraction of the expected supernova mechanical energy input, resulting in a "missing energy" problem. In late-type galaxies that are rich in cool gas much of this missing energy is possibly radiated in the UV band. This is supported by recent FUV emission-line observations (Otte et al.2003), which indicate that emission in the two O VI lines at $\lambda \lambda 1031.9,1037.6$ may play an important role for the overall energy balance in the hot gas. In collisional ionization equilibrium, O VI traces hot gas at temperature near $300,000 \mathrm{~K}$, thus at the peak of the cooling curve of (metalenriched) interstellar gas. At these temperatures, $\mathrm{O}$ VI is expected to be the dominant coolant in the gas, and thus O VI-inferred cooling most likely can account for a considerable fraction of the initial supernova energy input. However, as observations in our own halo indicate (e.g., Fox et al. 2004; Section 3.3 ), O VI emission may preferentially arise in the interface regions between the million-degree coronal gas and the neutral IVC and HVC gas. Additional observations will be required to learn more about the distribution and physics of the $\mathrm{O}$ VI emission in the halos of galaxies. Establishing a reliable estimate of the radiative cooling rate and unveiling the physical connection between FUV and X-ray emission will be of great importance to understand the physical conditions in the hot extraplanar gas of galaxies. 


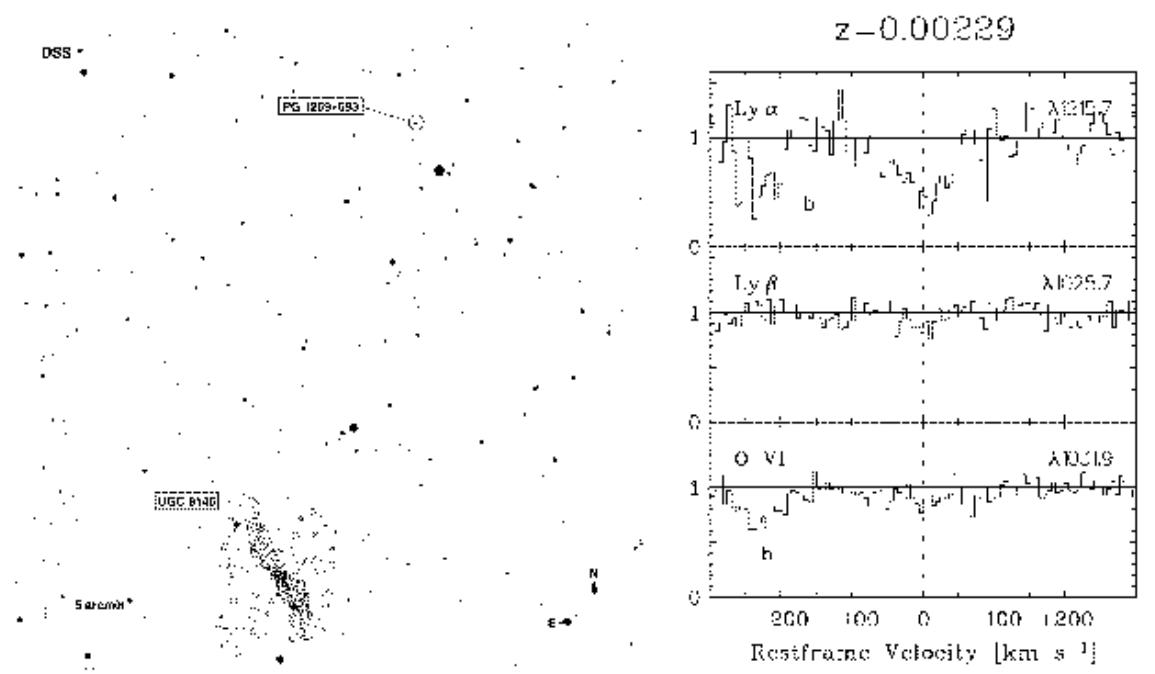

Figure 7: Left panel: sky positions of the quasar PG $1259+593$ and the galaxy UGC $08146(z=0.00224)$ on an image from the Digitized Sky Survey. H I contours for UGC 08146 from data of the Westerbork Synthesis Radio Telescope (Rhee \& van Albada 1996) are overlaid. Right panel: H I and O VI absorption profiles of the $z=0.00229$ absorption system toward PG $1259+593$. The absorption probably arises from gas in the halo or immediate intergalactic environment of UGC 08146. Figure adopted from Richter et al. (2004). [PLEASE CONTACT THE AUTHOR FOR A HIGH-QUALITY VERSION OF THIS FIGURE.]

\subsection{Circumgalactic Absorption-Line Systems}

The analysis of strong $(\log N(\mathrm{H} \mathrm{I}) \geq 15)$ quasar absorption-line systems that have associated metal lines (metal-line systems) is another important diagnostic tool to investigate the halos of galaxies and their intergalactic neighborhood at low and high redshifts. The presence of intervening absorptionline systems in QSO spectra can be understood in terms of intergalactic gas filaments that trace baryon density fluctuations related to gravitational instability during the large-scale structure formation in the Universe (see next Section). In this context, absorption-line systems that have neutral gas column densities in the range $\log N(\mathrm{H} \mathrm{I})=15-19$ represent prime candidates for gaseous structures that reside in the circumgalactic environment of galaxies: the neutral gas column densities of metal-line systems are clearly higher than in the photoionized, low-density intergalactic medium, but they are lower than characteristic column densities in the gaseous disks of present-day galaxies.

To find out whether or not an individual metal-line absorber in a QSO spectrum is related to the halo of a galaxy, one first has to identify candidate 
galaxies in the field of the background quasar. In a second step, one has to compare the absorber redshift with the redshift of the candidate galaxy to identify true (i.e., physically connected) galaxy/absorber pairs. Using this technique, many galaxy/absorber pairs have been found, implying that galaxy halos extend far into intergalactic space, leaving their imprint in the spectra of nearby (in terms of angular separation) quasars. A particularly good tracer of metal-enriched gas that is associated with galaxy halos at low redshift is the strong resonant Mg II $\lambda \lambda 2796.4,2803.5$ doublet in absorption (e.g., Bergeron \& Stasinska 1986; Churchill et al.1999; Churchill et al. 2005). As an $\alpha$-process element, magnesium is ejected into the interstellar and intergalactic gas by supernova explosions and thus should be abundant in the halos of star-forming galaxies at low redshift. In addition, Mg II arises in gas that spans roughly five orders of magnitudes in total gas column densities (log $N(\mathrm{H} \mathrm{I}) \approx 15.5-20.5)$ and thus is a sensitive tracer for circumgalactic gas that may have very different ionization fractions and physical conditions. Several studies of Mg II absorbers in the fields of (absorption-selected) low-redshift galaxies have unveiled the connection between the galaxy luminosities, colors, and the extent of the Mg II absorbing envelopes around galaxies (e.g., Bergeron \& Boissé 1991; Steidel et al. 1994). In general, the luminosities of Mg II selected galaxies show only little evolution with redshift, while the sizes of the Mg II halos scale only weakly with the luminosities of the galaxies (see, e.g., Churchill et al.2005). These measurements have shown that the gas cross section for strong $\mathrm{Mg}$ II absorbers with equivalent widths $(\mathrm{EWs})>0.3 \AA$ is $\sim 40 \mathrm{kpc}$, whereas for weaker systems with EWs $\leq 0.3 \AA$ the cross section is $\sim 70 \mathrm{kpc}$. These values characterize the sizes of $\mathrm{Mg}$ II halos of galaxies (that may have quite different morphologies). Note that the decrease of the Mg II equivalent width with increasing distance to the galaxy does not necessarily indicate a diminishing total gas column density. It rather implies that the ionization fraction of the gas is higher at larger distances, as shown by observations of other, highly-ionized species. It is therefore important to also consider other ions (e.g., C IV and/or O vi together with $\mathrm{H}$ I) for absorptionline studies of galaxy halos and the circumgalactic gaseous environment of galaxies.

As an example, we show in Fig. $7 \mathrm{H} \mathrm{I}$ and O vi absorption at $z=0.00229$ in the spectrum of the quasar PG $1259+593\left(z_{\mathrm{em}}=0.478\right)$, based on FUSE observations (Richter et al. 2004). This sightline lies close to the dwarf spiral galaxy UGC 08146, which has a redshift of $z=0.00224$. In Fig. 7, left panel, we show the sky positions of PG $1259+593$ and UGC 08146 on an image taken from the Digitized Sky Survey (DSS). We have overlaid H I $21 \mathrm{~cm}$ contours from data of the Westerbork Synthesis Radio Telescope (WSRT; from Rhee \& von Albada 1996). Assuming $H_{0}=75 \mathrm{~km} \mathrm{~s}^{-1} \mathrm{Mpc}^{-1}$, the redshift of $z=0.00224$ $\left(672 \mathrm{~km} \mathrm{~s}^{-1}\right)$ corresponds to a distance of $\sim 9.0 h_{75}^{-1} \mathrm{Mpc}$. Five arcmin in Fig. 7 therefore are equivalent to $\sim 13 \mathrm{kpc}$ at the distance of UGC 08146 . The angular separation between UGC 08146 and the line of sight towards $\mathrm{PG} 1259+593$ is $\sim 21$ arcmin, so that the projected distance is $\sim 55 h_{75}^{-1}$ kpc. The $\mathrm{H}$ I and O VI absorption at $z=0.00229$ in the spectrum of the 
quasar PG $1259+593$ therefore most likely arises in the halo or immediate intergalactic gaseous environment of UGC 08146.

The absorption-line characteristics of absorption-line systems around galaxies in the low-redshift Universe can be compared with absorption line systems arising the halo of our own galaxy. While most of the absorption measurements in the halo of the Milky Way concentrate on the diagnostics of $\mathrm{HI}$ selected halo clouds (i.e., IVCs and HVCs), Richter et al. (2005) have investigated Ca II absorption in optical spectra of quasars that are distributed randomly in the sky. Although the optical Ca II lines are less sensitive than the strong Mg II transition in the UV, they can be used to trace neutral highvelocity gas structures in the Milky Way halo at total gas column densities below the detection limit of the large H i HVC surveys (e.g., the LAB survey, Kalberla et al. 2005). The big advantage of using Ca II absorption is that large ground-based telescopes such as the Very Large Telescope (VLT) can be used for such studies. Therefore, a large number of high $\mathrm{S} / \mathrm{N}$, high resolution spectra through the halo are available to study Ca II absorption in the Galactic halo at high accuracy. In contrast, Mg II and C IV studies of gas in the Galactic halo are limited in number and accuracy due to the restricted sensitivity and availability of space-based UV spectrographs. From a preliminary analysis of VLT/UVES spectra, Richter et al. (2005) find that weak Ca II absorption at high-velocities is seen in more than $\sim 50$ percent of the QSO spectra, also in directions where no corresponding $\mathrm{H} \mathrm{I} 21 \mathrm{~cm}$ emission is seen in the large HVC $21 \mathrm{~cm}$ surveys (e.g., Kalberla et al. 2005). This implies that - next to the well-known high-column density HVCs - the halo is filled with low-column density neutral gas clouds that possibly represent the local counterparts of weak circumgalactic Mg II systems at low redshift that have equivalent widths of $\leq 0.3 \AA$.

\section{The Local Intergalactic Medium}

\subsection{Overview}

Shortly after the first detection of quasars in the early 1960s, the occurrence of many narrow absorption lines in a QSO spectrum was recognized for the first time (see, e.g., Bahcall 1966). It soon became clear that these absorption lines are related to intervening gaseous structures that fill the intergalactic space. These structures represent the intergalactic medium (IGM).

By far most of the intervening absorption lines in QSO spectra are produced by $\mathrm{H}$ I absorption in the Ly $\alpha$ line $\left(\lambda_{0}=1215.67 \AA\right)$, which is redshifted to higher wavelengths by the factor $(1+z)$, where $z$ denotes the redshift of an individual intergalactic absorber. With the availability of high-resolution $\mathrm{N}$ body hydrodynamical models (e.g., Davé et al. 2001) the Ly $\alpha$ absorbers have been interpreted as gaseous structures that arise from baryon density fluctuations associated with gravitational instability during the large-scale structure formation in the Universe. While the few strong absorption-line systems with 
H I column densities $>10^{15} \mathrm{~cm}^{-2}$ (and associated metal absorption) are believed to trace condensed structures such as extended ionized galaxy halos, protogalaxies, and gaseous disks of galaxies, the vast majority of the intervening absorption lines in QSO spectra are weak $\left(N(\mathrm{H} \mathrm{I})<10^{15} \mathrm{~cm}^{-2}\right)$. In fact, the $\mathrm{H}$ I column-density distribution function of QSO absorption-line systems at high redshift is (in a first-order approximation) a power-law that scales with $N(\mathrm{H} \mathrm{I})^{-1.5}$ (e.g., Petitjean et al. 1993). Most of the weak intervening $\mathrm{H}$ i lines belong to the so-called "Ly $\alpha$ forest", which predominantly traces extended, highly-ionized intergalactic structures - the "true" intergalactic medium. This gas has very low volume densities $\left(n_{\mathrm{H}}<10^{-4} \mathrm{~cm}^{-3}\right.$, typically) and is photoionized by the ambient UV background radiation from quasars and active galactic nuclei. As structure evolution in the Universe proceeds from high to low redshift, however, an increasing fraction of the IGM is expected to be shock-heated and collisionally ionized, as the medium is collapsing under the action of gravity in the deeper potential wells of the condensing large-scale structure. Since the intergalactic medium is highly ionized (the neutral gas fraction is typically far less than a percent) it is clear that the observed weak neutral hydrogen absorption serves only as a sign for large amounts of ionized gas that is distributed in a filamentary network all over intergalactic space.

Following the results from IGM observations and numerical simulations, photoionized and collisionally ionized intergalactic gas most likely makes up for most of the baryonic matter in the local Universe (where $\Omega_{b} \approx 0.045$ ). While the diffuse photoionized IGM that gives rise to the Lyman $\alpha$ forest accounts for $\sim 30$ percent of the baryons today (Penton, Stocke, \& Shull 2004), the shock-heated warm-hot intergalactic medium at temperatures $T \sim$ $10^{5}-10^{7} \mathrm{~K}$ is expected to contribute at a comparable level to the cosmological mass density of the baryons at $z=0$ (Cen \& Ostriker 1999; Davé et al. 2001). Gas and stars in galaxies, groups of galaxies, and galaxy clusters make up the rest of the baryonic mass (Fukugita 2003).

\subsection{The Photoionized Ly $\alpha$ Forest at Low Redshift}

Absorption-line studies of the IGM at high redshift $(z>1.5)$ represent an essential observational method to directly study the structure evolution in the early Universe. Since all the important UV lines are redshifted into the optical band, absorption-line spectroscopy of the high- $z$ IGM with $8-10 \mathrm{~m}$ class telescopes and state-of-the art spectrographs provide excellent spectral data, i.e., spectra with high $\mathrm{S} / \mathrm{N}$ ratios at high spectral resolution. In contrast, measurements of the IGM at low $z$ require space-based UV satellites and thus are much more challenging than for high $z$. This fact leads to the somewhat unsatisfying situation that many aspects of the local IGM and the IGM evolution from high to low redshifts are not well understood yet.

As measurements show, the number density (per unit redshift) of Ly $\alpha$ absorbers at high $z(1.5 \leq z \leq 4.0)$ evolves rapidly, $d N / d z \propto(1+z)^{\gamma}$, where $\gamma \approx 2.2$ (e.g., Kim et al. 2001). With the availability of HST, it therefore was a surprise to find that at low redshifts $(z<1.5)$ the evolution appears 
to be nearly flat, with $\gamma \approx 0.1-0.3$ (Weymann et al.1998). Note that these early low- $z$ observations of the Ly $\alpha$ forest were based on data obtained with the Faint Object Spectrograph (FOS), which has relatively low spectral resolution. More recent, high-resolution HST/GHRS measurements (Penton et al. 2002) have shown, however, that the slowing of the Ly $\alpha$ density distribution in higher-resolution data is not as great as previously measured in lower-resolution data. The break to slower evolution probably occurs at $z \sim 1.0$ rather than at $z \sim 1.5$. From these high-resolution GHRS measurements it also follows that higher-column density Ly $\alpha$ forest systems tend to cluster more strongly with galaxies than low-column density systems (Penton et al. 2002). An example for a combined FUSE/STIS high-resolution low- $z$ IGM spectrum toward the quasar PG $1259+593$ is shown in Fig. 8.

The evolution of the Ly $\alpha$ forest density from high to low redshift can be understood in terms of the expansion of the Universe and the changing ionizing background flux. For redshifts from 5 to 1 the Ly $\alpha$ forest density is decreasing rapidly due to the expanding Universe and the only mildly decreasing ionizing flux. At $z<1$ the evolution is slowed down substantially due to the strongly decreasing ionizing background, which must have dropped by about one order of magnitude from $z=1$ to $z=0$ (Haardt \& Madau 1996). The line statistics together with a photoionization model of the Ly $\alpha$ forest implies that the warm, photoionized intergalactic medium at $z \approx 0$ contains $\sim 30$ percent of the baryons in the local Universe (Penton et al. 2004). For comparison, the contribution from the Ly $\alpha$ forest to the baryon density $\left(\Omega_{b}\right)$ at $z=2$ is $\sim 90$ percent. In the course of the structure evolution in the Universe from high to low redshifts the baryon budget in the Ly $\alpha$ forest therefore has dropped substantially. This is partly due to the formation and assembly of condensed galactic structures, but also due to the appearance of yet another intergalactic gaseous component, the warm-hot intergalactic medium (WHIM).

\subsection{The Warm-Hot Intergalactic Medium}

As the temperature of the intergalactic medium undergoes a significant change from high to low redshifts, a large fraction of the baryonic matter in the local Universe is expected to reside in the WHIM phase - a gas phase that is particularly difficult to detect. The WHIM is believed to emerge from intergalactic gas that is shock-heated to high temperatures as the medium is collapsing under the action of gravity (Valageas, Schaeffer, \& Silk 2002). Directly observing this gas phase is challenging, as the WHIM represents a low-density $\left(n_{\mathrm{H}} \sim 10^{-6}-10^{-4} \mathrm{~cm}^{-3}\right)$, high-temperature $\left(T \sim 10^{5}-10^{7}\right.$ $\mathrm{K}$ ) plasma that primarily is made of protons, electrons, $\mathrm{He}^{+}$, and $\mathrm{He}^{++}$, together with traces of some highly-ionized heavy elements. Diffuse emission from this plasma is expected to have a very low surface brightness and its detection awaits UV and X-ray observatories more sensitive than currently available (see, e.g., Fang et al. 2005; Kawahara et al. 2005). A promising approach to study the WHIM is the search for absorption features from the WHIM in FUV and in the X-ray regime. Five-times ionized oxygen (O vI) 


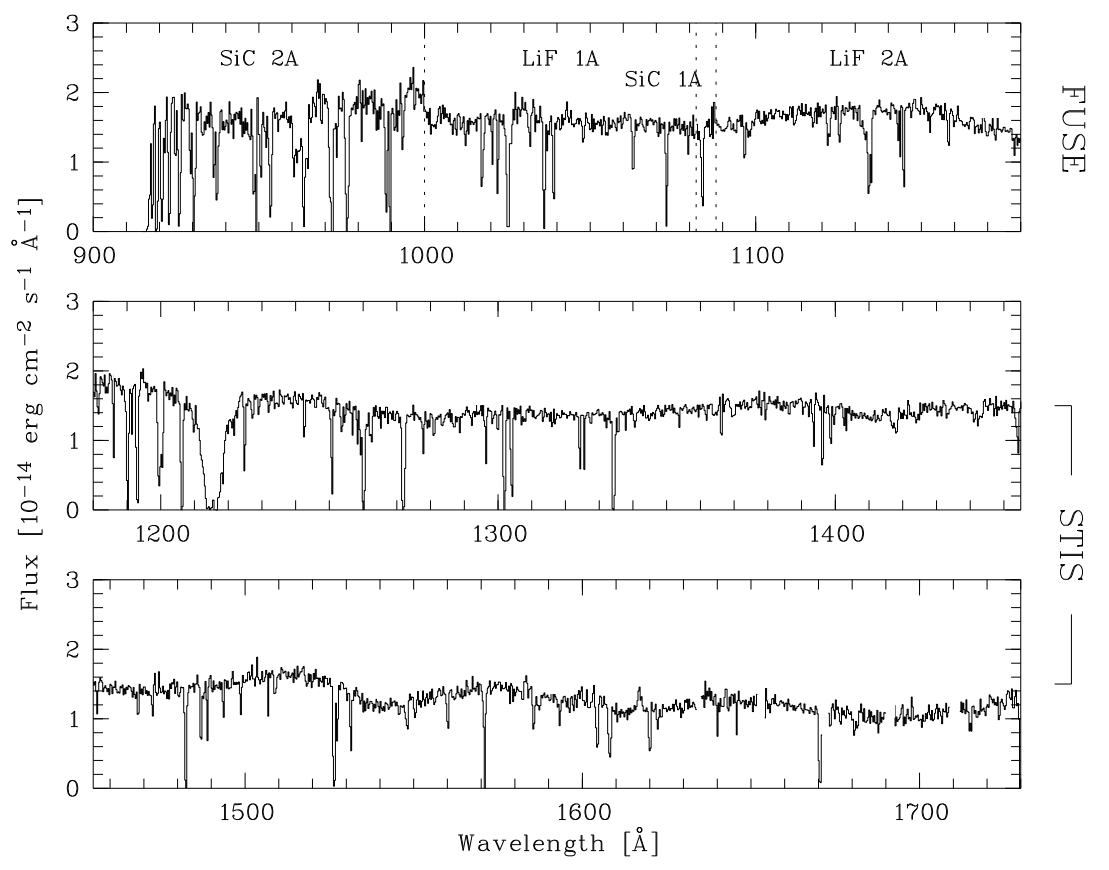

Figure 8: Combined FUSE and STIS spectrum of the low-redshift quasar PG $1259+593\left(z_{\mathrm{em}}=0.478\right)$ in the wavelength range between 900 and 1730 $\AA$. Next to absorption from gas in the Milky Way, the spectrum contains a number of low- $z$ intergalactic absorption lines, as discussed in Richter et al. (2004).

is the most important high ion to trace the WHIM at temperatures of $T \sim$ $3 \times 10^{5} \mathrm{~K}$ in the FUV regime. Oxygen is a relatively abundant element and the two available O vi transitions (located at 1031.9 and $1037.6 \AA$ ) have large oscillator strengths. A number of detections of intervening WHIM O VI absorbers at $z<0.5$ have been reported in the literature (Tripp, Savage, \& Jenkins 2000; Oegerle et al. 2000; Chen \& Prochaska 2000; Savage et al. 2002; Richter et al. 2004, Sembach et al. 2004b; Savage et al. 2005; Danforth \& Shull 2005 ). These measurements imply a number density of O VI absorbers per unit redshift of $d N_{\mathrm{OVI}} / d z \approx 17 \pm 3$ for equivalent widths $W_{\lambda} \geq 30 \mathrm{~m} \AA$ (Danforth \& Shull 2005). Assuming that 20 percent or less of the oxygen is present in the form of $\mathrm{O}$ VI $\left(f_{\mathrm{O} V \mathrm{I}} \leq 0.2\right)$ and further assuming a mean oxygen abundance of 0.1 solar, the measured number density of $\mathrm{O}$ VI absorbers corresponds to a cosmological mass density of $\Omega_{b}(\mathrm{O} \mathrm{vI}) \geq 0.0024 h_{75}{ }^{-1}$. Fig. 9 shows an example of an intervening $\mathrm{O}$ VI system at $z=0.31978$ in the direction of PG 1259+593. For the interpretation of $\Omega_{b}(\mathrm{O}$ VI $)$ it has to be noted that $\mathrm{O}$ VI absorption traces collisionally ionized gas at temperatures around $3 \times 10^{5}$ $\mathrm{K}$ (and also low-density, photoionized gas at lower temperatures), but not 
the million-degree gas phase which probably contains the majority of the baryons in the WHIM. Very recently, Savage et al. (2005) have reported the detection of Ne VIII in an absorption system at $z \approx 0.2$ in the direction of the quasar HE $0226-4110$. Ne VIII traces gas at $T \sim 7 \times 10^{5} \mathrm{~K}$ (in collisional ionization equilibrium) and thus is possibly suited to complement the $\mathrm{O}$ VI measurements of the WHIM in a higher temperature regime. However, as the cosmic abundance of Ne VIII is relatively low, Ne VIII is not expected to be a particularly sensitive tracer of the WHIM at the $\mathrm{S} / \mathrm{N}$ levels achievable with current UV spectrographs. This is supported by the non-detections of intervening Ne VIII in other high S/N STIS data (Richter et al. 2004). Also $\mathrm{X}$-ray absorption measurements are very important for studying the WHIM (e.g., Fang et al. 2002; Nicastro et al. 2005), but they currently are limited in scope because of the small number of available background sources and the relatively low spectral resolution of current X-ray observatories (FWHM $\sim 500$ to $1000 \mathrm{~km} \mathrm{~s}^{-1}$ ).

Next to high-ion absorption from oxygen and other metals, recent observations with STIS (Richter et al. 2004; Sembach et al.2004b) have shown that WHIM filaments can be detected in Ly $\alpha$ absorption of neutral hydrogen. Although the vast majority of the hydrogen in the WHIM is ionized (by collisional processes and UV radiation), a tiny fraction $\left(f_{\mathrm{H} \mathrm{I}}<10^{-5}\right.$, typically) of neutral hydrogen is expected to be present. Depending on the total gas column density of a WHIM absorber and its temperature, weak $\mathrm{H} \mathrm{I}$ Ly $\alpha$ absorption at column densities $12.5 \leq \log N(\mathrm{H} \mathrm{I}) \leq 14.0$ may arise from WHIM filaments and could be used to trace the ionized hydrogen component. The Ly $\alpha$ absorption from WHIM filaments is very broad due to thermal line broadening, resulting in large Doppler parameters of $b>40 \mathrm{~km} \mathrm{~s}^{-1}$. Such lines are generally difficult to detect, as they are broad and shallow. High resolution, high S/N FUV spectra of QSOs with smooth background continua are required to successfully search for broad Ly $\alpha$ absorption in the low-redshift WHIM. STIS installed on the HST is the only instrument that has provided such data, but due to the instrumental limitations of spacebased observatories, the number of QSO spectra adequate for searching for WHIM broad Ly $\alpha$ absorption (in the following abbreviated as "BLA") is very limited. An example for a BLA at $z=0.18047$ in the STIS spectrum of the quasar H $1821+643$ is shown in Fig. 10. So far, four sight lines observed with STIS towards the quasars PG $1259+593\left(z_{\mathrm{em}}=0.478\right)$, PG $1116+215$ $\left(z_{\mathrm{em}}=0.176\right), \mathrm{H} 1821+643\left(z_{\mathrm{em}}=0.297\right)$, and PG 0953+415 $\left(z_{\mathrm{em}}=0.239\right)$ have been carefully inspected for the presence of BLAs, and a number of good candidates have been identified (Richter et al. 2004, 2006a; Sembach et al. 2004). These measurements imply a BLA number density per unit redshift of $d N_{\mathrm{BLA}} / d z \approx 22-53$ for Doppler parameters $b \geq 40 \mathrm{~km} \mathrm{~s}^{-1}$ and above a sensitivity limit of $\log \left(N\left(\mathrm{~cm}^{-2}\right) / b\left(\mathrm{~km} \mathrm{~s}^{-1}\right)\right) \geq 11.3$. The large range for $d N_{\text {BLA }} / d z$ partly is due to the uncertainty about defining reliable selection criteria for separating spurious cases from good broad Ly $\alpha$ candidates (see discussions in Richter et al. 2004, 2006a and Sembach et al. 2004). Transforming the number density $d N_{\mathrm{BLA}} / d z$ into a cosmological baryonic mass density, 


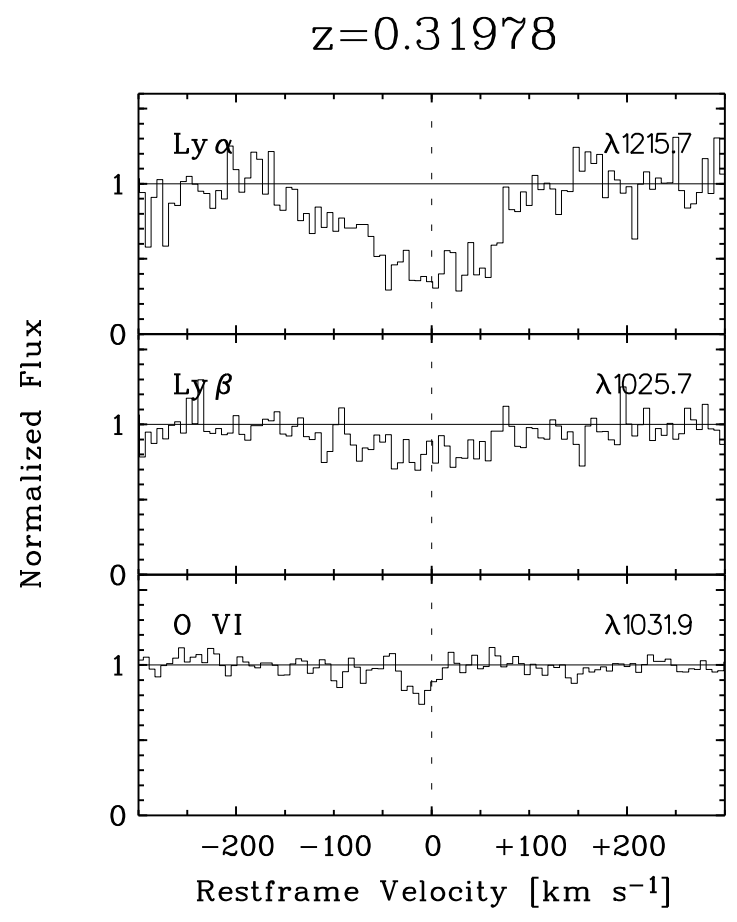

Figure 9: Normalized absorption profiles of $\mathrm{HI}$ and $\mathrm{O}$ VI in the $\mathrm{O}$ VI/BLA system at $z=0.31978$ toward PG $1259+593$. Both the occurrence of $\mathrm{O}$ VI absorption and the large width of the $\mathrm{HI}$ absorption (probably caused by thermal line broadening) are consistent with collisionally ionized gas at a temperature near $3 \times 10^{5} \mathrm{~K}$. The total gas column density in this system is estimated to be $\sim 6.4 \times 10^{19} \mathrm{~cm}^{-2}$. Figure and numbers taken from Richter et al. (2004).

one obtains $\Omega_{b}$ (BLA) $\geq 0.0029 h_{75}{ }^{-1}$. This limit is about 6 percent of the total baryonic mass density in the Universe expected from the current cosmological models (see above), and is slightly above the limit derived for the intervening O vi absorbers $\left(\Omega_{b}(\mathrm{O} v \mathrm{vI}) \geq 0.0024 h_{75}{ }^{-1}\right.$; see above). The analysis of BLAs in cosmological simulations (Richter et al. 2006b) supports the idea that these systems represent a huge baryon reservoir in the local Universe.

\subsection{Distribution of Metals}

As the many detections of metal lines in intervening absorption-line systems show, the intergalactic medium contains heavy elements. These elements must have been produced in stars within galaxies and then transported through an efficient mechanism into the IGM. The prime candidate for the metal enrichment of the IGM is outflows from starbursting dwarf galaxies (e.g., 


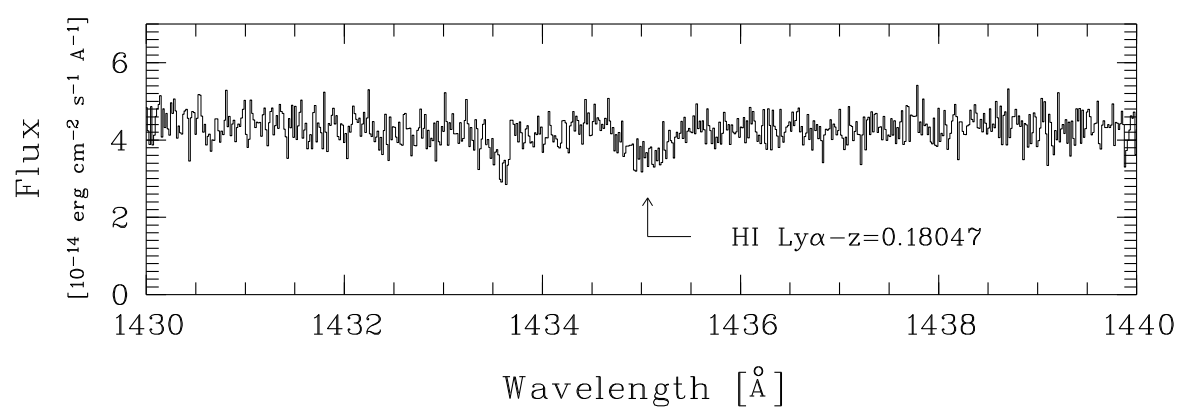

Figure 10: The well-detected broad Ly $\alpha$ absorber (BLA) at $z=0.18047$ in the STIS spectrum of $\mathrm{H} 1821+643$ is shown. The shape of this BLA differs significantly from the shape of the narrow lines from the Ly $\alpha$ forest, such as the $z=0.17924$ Ly $\alpha$ forest absorber near $1433.5 \AA$. Figure taken from Richter et al. (2005b).

Heckman et al. 2001). Recent studies using FUSE absorption-line data imply that the metallicity of the low- $z$ IGM is $\sim 0.1$ solar. For instance, the number density of intervening O VI systems observed toward low-redshift QSOs is in agreement with the predicted distribution of $\mathrm{O}$ VI absorbers in numerical simulations that assume an oxygen abundance of 0.1 solar (Danforth \& Shull 2005). Also the measurements of intergalactic C III together with appropriate multi-phase ionization models suggest a mean metallicity of the local IGM that is roughly ten percent solar (Danforth et al. 2005).

While all these studies aim at providing an accurate mean metallicity of the local IGM, it has to be kept in mind that metal abundances in individual intergalactic regions may deviate substantially from this mean IGM metallicity. An example for an O vi system with an oxygen abundance that probably is much less than 0.1 solar is the absorber at $z=0.31978$ towards the quasar PG 1259+593 (Richter et al. 2004; see Fig. 9). Due to its simplicity and its suggested high-temperature characteristics, this system represents an interesting case for a more detailed modeling of the physical conditions and the oxygen abundance. The large $\mathrm{H}$ I $b$ value and the presence of $\mathrm{O}$ VI already suggest that this absorption system consists mainly of hot gas at temperatures around $3 \times 10^{5} \mathrm{~K}$. Using the absorption width as a measure for the temperature of the gas, the collisional ionization model implies hat the $\mathrm{O}$ abundance in this $\mathrm{O}$ vi system is only $\sim 4.3 \times 10^{-3}$ solar. An abundance as low as $\sim 4 \times 10^{-3}$ solar, if correct, has important implications since the estimated baryonic content of the $\mathrm{O}$ vi systems scales inversely with the assumed oxygen abundance. Various groups (e.g., Savage et al.2002; Sembach et al.2004b; Danforth \& Shull 2005) have estimated that the gas in low redshift O vi systems contributes with $\sim 0.002$ to the cosmological closure density assuming that the average oxygen abundance in O vi systems is 0.1 solar. This contribution is comparable to that found in galaxies but $\sim 20$ times smaller than the total contribution for baryons estimated from the Cosmic Background radiation or 
from big bang nucleosynthesis. However, if the typical oxygen abundances in some of the $\mathrm{O}$ VI systems are 10 to 25 times smaller, the estimate for the baryonic content of these systems would increase by factors of 10 to 25. Finding low-metallicity regions in the local IGM and studying their ionization properties therefore is of great importance to provide reliable estimates of the baryon budget in WHIM O VI absorbers.

\section{Conclusions and Outlook}

The results discussed in this article demonstrate that studies of the distribution and physical properties of the gaseous circumgalactic and intergalactic environment of galaxies are important to understand the formation and evolution of individual galaxies and the large-scale structure formation in the Universe. The use of the absorption-line technique in the FUV and optical in combination with emission measurements at X-ray, UV, optical, and radio wavelengths provides a particularly powerful to study the gaseous environment of galaxies and the intergalactic medium at low redshift. Concerning our own galaxy, such combined absorption and emission measurements have shown that the formation of the Milky Way is still on-going. Other studies have demonstrated that the gaseous environment of galaxies and the intergalactic medium is (in general) governed by a complex interplay between gaseous infall and outflow and the hierarchical merging of (galactic) structures in the local Universe. Despite the low densities that characterize the gas in the outskirts of galaxies and in the intergalactic medium, it has become clear that (due to the large filling factor) this gas contains a substantial fraction of the baryonic mass in the local Universe.

\section{Acknowledgements}

First of all, I thank the Astronomische Gesellschaft for awarding me the Ludwig Biermann Price - I am deeply honored. Clearly, this price serves as an inspiration and motivation for my future scientific work. I particularly like to thank my former supervisors Klaas de Boer and Blair Savage, who greatly supported me over the last few years and from whom I learned so much. I am grateful to my other past and recent collaborators and supporting colleagues Jacqueline Bergeron, Hartmut Bluhm, Christian Brüns, Greg Bryan, Ralf-Jürgen Dettmar, Taotao Fang, Andrea Ferrara, Andrew Fox, Gerhard Hensler, Michael Hilker, Hiro Hirashita, Chris Howk, Peter Kalberla, Cedric Ledoux, Ole Marggraf, Patrick Petitjean, Tom Richtler, Peter Schneider, Ken Sembach, Todd Tripp, Bart Wakker, Tobias Westmeier, and many others. It is a great pleasure to work together with so many bright and inspiring scientists. Also, I would like to thank Klaas de Boer, Blair Savage, and Tobias Westmeier for a critical reading of the manuscript. Last but not least, I thank the Deutsche Forschungsgemeinschaft (DFG) for financial support in the DFG Emmy-Noether program. 


\section{References}

Bahcall, J.N. 1966, ApJ 145, 684

Bergeron, J., Stasinska, G. 1986, A\&A 169, 1

Bergeron, J., \& Boissé, P. 1991, A\&A 243, 344

Blitz, L., Spergel, D.N., Teuben, P.J., Hartmann, D., \& Burton, W.B. 1999, ApJ 514, 818

Bluhm, H., deBoer, K., Marggraf, O., \& Richter, P. 2001, A\&A 367, 299

Braun, R., \& Burton,, W.B. 1999, A\&A 341, 437

Braun, R., \& Burton, W.B. 2000, A\&A 354, 853

Brown, W.R., Geller, M.J., Kenyon, S.J., et al. 2004, AJ 127, 1555

Cen, R., \& Ostriker, J. 1999, ApJ 514, 1

Chen, H.-W., \& Prochaska, J.X. 2000, ApJ 543, L9

Christlieb, N., Beers, T.C., Barklem, P., et al. 2004, A\&A 428, 1027

Churchill, C.W., Rigby, J.R., Charlton, J.C., Vogt, S.S. 1999, ApJS 120, 51

Churchill, C.W., Steidel, C.C., Kacprzak, G. 2005, in Extra-planar gas, ed. R. Braun, ASP Conference Series, Vol. 331, 387

Collins, J.A., Shull, J.M., \& Giroux, M.L. 2003, ApJ 585, 336

Danforth, C.W., \& Shull, J.M. 2005, ApJ 624, 555

Danforth, C.W., Shull, J.M., Rosenberg, J.L., \& Stocke, J.T. 2005, astro-ph 0508656

Davé, R., Cen, R., Ostriker, J., et al. 2001, ApJ 552, 473

de Boer, K.S. 2004, A\&A 419, 527

de Boer, K.S. 2004, in High-velocity Clouds, ed. H. van Woerden et al., ASSL, Vol. 312 (Kluwer), ISBN 1402025785, 227

Fang, T., Marshall, H.L., Lee, J.C., Davis, D.S., \& Canizares, C.R. 2002, ApJ 572 L127

Fang, T., Croft, R.A.C., Sanders, W.T., et al. 2005, ApJ 623, 612

Fox, A.J., Savage, B.D., Wakker, B.P., et al. 2004, ApJ 602, 738

Fukugita, M. 2003, astro-ph 0312517

Gringel, W., Barnstedt, J., de Boer, K.S., et al. 2000, A\&A 358, L37

Haardt, F., \& Madau, P. 1996, ApJ 461, 20

Hartmann, D., \& Burton, W.B. 1997, Atlas of Galactic Neutral Hydrogen, Cambridge University Press

Heckman, T.M., Sembach, K.R., Meurer, G.R., et al. 2001, ApJ 554, 1021

Heiles C. 1997, ApJ 481, 193

Heithausen, A. 2004, ApJ 606, L13

Howk, J.C., \& Savage, B.D. 1997, AJ 114, 2463

Howk, J.C., \& Savage, B.D. 1999, AJ 117, 2077

Howk, J.C., \& Savage, B.D. 2000, AJ 119, 644 
Houck, J.C., \& Bregman, J.N. 1990, ApJ 352, 506

Hulsbosch, A.N.M., Wakker, B.P. 1988, A\&AS 75, 191

Ibata, R.A., Gilmore, G., \& Irwin, M.J. 1994, Nature 370, 194

Kalberla, P. M. W., Burton, W. B., Hartmann, D., et al. 2005, A\&A 440, 775

Kawahara, H., Yoshikawa, K., Sasaki, S., et al. 2005, astro-ph 0504594

Kim, T.-S., Christiani, S., \& D'Odorico, S. 2001, A\&A 373, 575

Lu, L., Sargent, W.L.W., Savage, B.D., et al. 1998, AJ 115, 162

Meyer D.M. \& Lauroesch J.T. 1999, ApJ 520, L103

Morras, R., Bajaja, E., Arnal, E.M., \& Pöppel, W.G.L. 2000, A\&AS 142, 25

Muller, C.A., Oort, J.H., \& Raimond, E. 1963, C.R. Acad. Sci. Paris 257, 1661

Münch, G. 1952, PASP 64, 312

Münch, G., \& Zirin, H. 1961, ApJ 133, 11

Murphy, E.M., Lockman, F.J., \& Savage, B.D. 1995, ApJ 447, 642

Nicastro, F., Zezas, A., Elvis, M., et al. 2003, Nature 421, 719

Nicastro, F., Mathur, S., Elvis, M., al. 2005, Nature 433, 495

Oegerle, W.R., Tripp, T.M., Sembach, K.R., et al. 2000, ApJ 538, L23

Oort, J.H. 1970, A\&A 7, 381

Oosterloo, T. 2004, in High-velocity Clouds, ed. H. van Woerden et al., ASSL, Vol. 312 (Kluwer), ISBN 1402025785, 125

Otte, B., Murphy, E.M., Howk, J.C., et al. 2003, ApJ 591, 821

Penton, S.V., Stocke, J.T., \& Shull, J.M. 2004, ApJS 152, 29

Penton, S.V., Stocke, J.T., \& Shull, J.M. 2002, ApJ 565, 720

Petitjean, P., Webb, J.K., Rauch, M., Carswell, R. F., \& Lanzetta, K. 1993, MNRAS 262, 499

Putman, M.E., Bland-Hawthorn, J., Veilleux, S., et al. 2003, ApJ 597, 948

Rand, R.J. 1996, ApJ 462, 712

Rhee, M.-H., \& van Albada, T.S. 1996, A\&AS 115, 407

Richter, P., de Boer, K.S., Widmann, H., et al. 1999, Nature 402, 386

Richter, P., Savage, B.D., Wakker, B.P., Sembach, K.R., Kalberla, P.M.W. 2001a, ApJ 549, 281

Richter, P., Sembach, K.R., Wakker, B.P., et al. 2001b, ApJ 559, 318

Richter P., Sembach K.R., Wakker B.P., \& Savage B.D. 2001c, ApJ 562, L181

Richter, P., Wakker, B.P., Savage, B.D., Sembach, K.R. 2003a, ApJ 586, 230

Richter, P., Sembach, K.R., Howk, J.C. 2003b, A\&A 405, 1013

Richter, P., \& de Boer, K.S. 2004, in High-velocity Clouds, ed. H. van Woerden et al., ASSL, Vol. 312 (Kluwer), ISBN 1402025785, 183

Richter, P., Savage, B.D., Tripp, T.M., \& Sembach, K.R. 2004, ApJS, 153, 165

Richter, P., Westmeier, T., \& Brüns, C. 2005, A\&A 442, L49 
Richter, P., Savage, B.D., Sembach, K.R., \& Tripp, T.M. 2006a, A\&A 445, 827

Richter, P., Fang, T., \& Bryan, G,L. 2006b, A\&A, in press; astro-ph 0511609

Rossa, J., \& Dettmar, R.-J. 2003, A\&A 406, 493

Savage, B.D., \& de Boer, K.S. 1979, ApJ 230, L77

Savage, B.D., \& de Boer, K.S. 1981, ApJ 244, 768

Savage, B.D., Sembach, K.R., \& Lu, L. 1997, AJ 113, 2158

Savage, B.D., Sembach, K.R., Tripp, T.M., \& Richter, P. 2002, ApJ 564, 631

Savage, B.D., Sembach, K.R., Wakker, B.P., et al. 2003, ApJS 146, 125

Savage, B.D., Lehner, N., Wakker, B.P., Sembach, K.R, \& Tripp, T.M. 2005, ApJ 626, 776

Sembach, K.R., Savage, B.D., \& Massa, D. 1991, ApJ 372, 81

Sembach, K.R., \& Savage, B.D. 1992, ApJS 83, 147

Sembach K.R., Howk J.C., Savage B.D., \& Shull J.M. 2001, AJ 121, 992

Sembach K.R., Wakker, B.P., Savage, B.D., et al. 2003, ApJS 146, 165

Sembach, K.R., Wakker, B.P., Tripp, T.M., et al. 2004a, ApJS 150, 387

Sembach, K.R., Tripp, T.M., Savage, B.D., \& Richter, P. 2004b, ApJS 155, 351

Shapiro, P.R., \& Field, G.B. 1976, ApJ 205, 762

Sirko, E., Goodman, J., Knapp, G.R., et al. 2004, AJ 127, 899

Spitzer, L. 1956, ApJ 124, 20

Steidel, C.C, Dickinson, M., \& Persson, E. 1994, ApJ 437, L75

Strickland, D.K., Heckman, T.M., Colbert, E.J.M., Hoopes, C.G., Weaver, K.A. 2004, ApJ 606, 829

Thilker, D.A., Braun, R., Walterbos, R.A.M., et al. 2004, ApJ 601, L39

Thom, C., Putman, M.E., \& Gibson, B.K. 2005, ApJL, in press

Tripp, T.M., Savage, B.D., \& Jenkins, E.B. 2000, ApJ 534, L1

Tripp, T.M., Wakker, B.P., Jenkins, E.B., et al. 2003, AJ 125, 3122

Valageas, P., Schaeffer, R., \& Silk J. 2002, A\&A 388, 741

van Woerden, H., Schwarz, U. J., Peletier, R. F., Wakker, B. P., \& Kalberla, P. M. W. 1999, Nature 400, 138

Wannier, P., \& Wrixon, G.T. 1972, ApJ 173, L119

Weiner, B.J., Vogel, S.N., Williams, T.B. 2001, in ASP Conf.Ser.240, Gas and Galaxy Evolution, ed. J.E. Hibbard, M.Rupen \& J. van Gorkom, 515

Wakker, B.P., Howk, J.C., Savage, B.D., et al. 1999, Nature, 402, 388

Wakker, B.P. 2001, ApJS 136, 463

Wakker, B.P., Savage, B.D., Sembach, K.R., et al. 2003, ApJS 146, 1

Wakker, B.P. 2004, in High-velocity Clouds, ed. H. van Woerden et al., ASSL, Vol. 312 (Kluwer), ISBN 1402025785, 25

Wakker, B.P., \& Richter, P. 2004, Scientific American 290, 28 
Wang, Q.D., Immler, S., Walterbos, R., Lauroesch, J.T.; Breitschwerdt, D. 2001, ApJ 555, L99

Wang, Q.D. 2005, in Extra-planar gas, ed. R. Braun, ASP Conference Series, Vol. 331, 329

Westmeier, T., Brüns, C., \& Kerp, J. 2005a, in Extra-planar gas, ed. R. Braun, ASP Conference Series, Vol. 331, 105

Westmeier, T., Braun, R., \& Thilker, D. 2005b, A\&A 436, 101

Weymann, R., Jannuzi, B.T., Lu, L., et al. 1998, ApJ 506, 1

Widmann, H., de Boer, K. S., Richter, P., et al. 1998, A\&A 338, L1

Wolfire, M.G., McKee, C.F., Hollenbach, D., \& Thielens, A.G.G.M. 1995, ApJ, 453, 673 